\title{
Noncritical anisotropic Bianchi type I string cosmology with $\alpha^{\prime}$ corrections
}

\author{
F. Naderi" \\ Young Researchers and Elite Club, Marand Branch, Islamic Azad University, Marand, Iran \\ A. Rezaei-Aghdam ${ }^{\dagger}$ and F. Darabi ${ }^{\ddagger}$ \\ Department of Physics, Faculty of Basic Sciences, Azarbaijan Shahid Madani University, \\ 53714-161 Tabriz, Iran
}

(Received 22 February 2018; published 5 July 2018)

\begin{abstract}
We present noncritical Bianchi type I string cosmology solutions in the presence of central charge deficit term $\Lambda$. The leading-order string frame curvature appears to be in the high curvature limit $R \alpha^{\prime} \gtrsim 1$, which underlines the necessity of including higher-order $\alpha^{\prime}$ corrections. We give new solutions of two-loop (firstorder $\alpha^{\prime}$ ) $\beta$-function equations of the $\sigma$ model with nonzero $\Lambda$ and the dilaton field in both cases of the absence and presence of a spatially homogeneous $H$ field $(H=d B)$. Also, the evolution of solutions is studied in the Einstein frame, in which the string effective action can transform to Gauss-Bonnet gravity model coupled to a dilaton field with potential. We study explicit examples in first-order $\alpha^{\prime}$ with chosen values of appeared constants in the solutions and discuss the cosmological implications.
\end{abstract}

DOI: 10.1103/PhysRevD.98.026009

\section{INTRODUCTION}

In the $\sigma$-model context, the conformal invariance is provided by vanishing $\beta$ functions [1], which are equivalent to the equations of motion of effective action in the string frame [2]. The low-energy string effective action, being compatible with the conformal invariance in one-loop order, has wide cosmological implications for describing the evolution of early Universe with a very low curvature and string coupling, $g_{s}=e^{-\phi}$ [3-5]. In two-loop order of $\beta$ functions, the string effective action is modified by including the $\alpha^{\prime}$ corrections of quadratic curvature type $\alpha^{\prime} R^{2}$, where the $\alpha^{\prime}$ is the square of the string length, $\alpha^{\prime}=\lambda_{s}^{2} / 2 \pi$ $[2,6]$. The expanded leading-order effective action is widely believed to regularize the curvature singularity [7]. The two-loop $\beta$ functions, possible $\alpha^{\prime}$-corrected string effective actions, and on-shell compatibility of the $\alpha^{\prime}$-corrected effective action equations of motion with the two-loop conformal invariance condition have been investigated in Ref. [2]. A renormalization scheme (RS) dependence appears in the $B$ field-dependent terms of two-loop $\beta$ functions and consequently in the $\alpha^{\prime}$-corrected effective

\footnotetext{
f.naderi@azaruniv.ac.ir †rezaei-a@azaruniv.ac.ir

f.darabi@azaruniv.ac.ir
}

Published by the American Physical Society under the terms of the Creative Commons Attribution 4.0 International license. Further distribution of this work must maintain attribution to the author(s) and the published article's title, journal citation, and DOI. Funded by SCOAP. actions, the two schemes of Gauss-Bonnet and $R^{2}$ of which have been considered distinctly in Ref. [2]. Furthermore, the $S$ matrix is invariant under a set of field redefinitions [8], which allows one to transform between RSs and leads to a physically equivalent class of effective actions [9].

Generally, two kinds of corrections can be included in the string effective action, the stringy $\alpha^{\prime}$ expansion and the quantum nature loop expansion in string coupling [5]. The $\alpha^{\prime}$ corrections are significant when the curvature is in the high limit $R \alpha^{\prime} \gtrsim 1$, while the loop corrections become important in the case of strong string coupling $g_{s}>1$. As long as the coupling is sufficiently weak in the high curvature regime, the $\alpha^{\prime}$ corrections are enough to be taken into account, and the loop corrections can be neglected [5].

Solutions of one-loop $\beta$-function equations with the contribution of the dilaton field and antisymmetric $B$ field have been presented for several cosmological backgrounds such as homogeneous anisotropic space-times [10-14] and inhomogeneous models [15]. According to Ref. [12], the contribution of the field strength tensor $H=d B$ in all Bianchi-type models with diagonal metrics can be classified into three classes of $\chi(\rightarrow), \chi(\uparrow)$, and $\chi(\nearrow)$, where the $\chi$ is all possible Bianchi types and the arrows indicate the orientation of $H^{*}$, the dual of $H$ with respect to the threedimensional (3D) hypersurface of homogeneity $\Sigma^{3}$ sections. In the aforementioned works, the central charge deficit term $\Lambda$ has been considered to be zero. In $D$ dimensions, $\Lambda$ is proportional to $D-26$ in bosonic string theory and $D-10$ in superstring theory and provides a term in the effective action analogous to the nonvanishing cosmological constant term [16]. Solutions with nonzero $\Lambda$, 
called noncritical string cosmology, have been obtained in the lowest-order $\beta$-function equations [16-18].

Moreover, neglecting the $\Lambda$ term, the solutions of twoloop $\beta$-function equations have been presented in some works, such as on the Kasner and Schwarzschild background with setting the $H$ field to zero [19] and on anisotropic homogeneous backgrounds with the contribution of the $H$ field [20]. Alternatively, the $\alpha^{\prime}$-corrected field equations of the effective action in the Einstein frame have been solved in several classes of backgrounds such as $\mathrm{M}$ theory, black holes, and cosmology with no contribution of the $H$ field [21-29]. Also, the solutions in the presence of the $H$ field have been investigated, for instance, on Friedmann-Robertson-Walker (FRW) and GarfinkleHorowitz-Strominger (GHS) black hole backgrounds with zero $\Lambda$ in Refs. [27,28] and on Godel space-time with contribution of $\Lambda$ in Ref. [29].

Especially, attempts to find accelerated expanding universes in the context of higher-dimensional superstring and $M$ theory led people to consider the extended gravitational actions, since, in the low-energy limit of their effective field theory, where the gravitational action is given only by the Einstein-Hilbert action, the accelerated expanding solutions are not allowed with a time-independent internal space [30]. In these theories, inflation is expected to occur at the Planck scale of 10 or 11 dimensions, and in such a highenergy scale, the higher-order corrections are required to be taken into account, at least in the early times. In this sense, accelerated solutions have been found in higher-order corrected high-dimensional string and $M$ theory in the absence of the $H$ field, for example, in Refs. [31-35], with special attention paid to the de Sitter-like and power-law expanding solutions.

In this work, aimed at presenting a noncritical fourdimensional two-loop string cosmology, we study the solutions of two-loop $\beta$-function equations on anisotropic Bianchi type I space-time with a nonzero $\Lambda$ and dilaton field in two cases of the presence and absence of an $H$ field. As we will show, the leading-order solutions have string frame curvature in the high limit $R \alpha^{\prime} \gtrsim 1$, where the higherorder $\alpha^{\prime}$ corrections become significant. We will limit our calculations to the first order in $\alpha^{\prime}$, in which the corrections of quadratic curvature types are included in the effective action. Concerning only this order of corrections, the regularizing effects of $\alpha^{\prime}$ corrections, in which the higher-order corrections usually act to correct the lowerorder solutions order by order, are already known $[5,21,25,27]$. We can, therefore, hope to provide a glimpse of the feature that could be obtained considering all orders in $\alpha^{\prime}$. Similar to what we have done in Ref. [20] for classifying and solving the two-loop $\beta$ function on all Bianchi-type models with $\Lambda=0$ and the $H$ field in the $\chi(\uparrow)$ class, a perturbative series expansion in $\alpha^{\prime}$ is implemented on the background field, and the general forms of equations and solutions are presented. Also, we consider the field equations in the Einstein frame by obtaining the contribution of $H$ field in $\alpha^{\prime}$ order of the energy-momentum tensor to investigate the cosmological implications of the $\alpha^{\prime}$ corrected solutions.

The paper is organized as follows. In Sec. II, the general forms of two-loop $\beta$ functions considering the two RS of Gauss-Bonnet and $R^{2}$ are recalled. Also, the field equations of the Gauss-Bonnet scheme in the Einstein frame are presented. In Sec. III, the two-loop $\beta$ functions with nonzero $\Lambda$ are solved on Bianchi type I background in the two cases of a vanishing and nonvanishing $H$ field. Then, the behavior of solutions is investigated in the Einstein frame in Sec. IV. Finally, the main results are summarized in Sec. V.

\section{TWO-LOOP (ORDER $\left.\alpha^{\prime}\right) \beta$ FUNCTIONS AND $\alpha^{\prime}$-CORRECTED STRING EFFECTIVE ACTION}

In a $\sigma$ model with background fields of metric $g$, dilaton field $\phi$, and antisymmetric $B$ field, the two-loop $\beta$ function of the metric is given by [2]

$$
\begin{aligned}
\frac{1}{\alpha^{\prime}} \beta_{\mu \nu}^{g}= & R_{\mu \nu}-\frac{1}{4} H_{\mu \nu}^{2}-\nabla_{\mu} \nabla_{\nu} \phi+\frac{\alpha^{\prime}}{2}\left[R_{\mu \alpha \beta \gamma} R_{\nu}^{\alpha \beta \gamma}-\frac{3}{2} R_{(\mu}^{\alpha \beta \gamma} H_{\nu) \alpha \lambda} H_{\beta \gamma}{ }^{\lambda}-\frac{1}{2} R^{\alpha \beta \rho \sigma} H_{\mu \alpha \beta} H_{\nu \rho \sigma}+\frac{1}{8}\left(H^{4}\right)_{\mu \nu}-\frac{f}{2}\left(R_{\mu \alpha \beta \nu}\left(H^{2}\right)^{\alpha \beta}\right.\right. \\
& \left.+2 R_{(\mu}^{\alpha \beta \gamma} H_{\nu) \alpha \lambda} H_{\beta \gamma}{ }^{\lambda}+R^{\alpha \beta \rho \sigma} H_{\mu \alpha \beta} H^{\nu \rho \sigma}-\nabla_{\lambda} H_{\mu \alpha \beta} \nabla^{\lambda} H_{\nu}^{\alpha \beta}\right)-\frac{1}{12} \nabla_{\mu} \nabla_{\nu} H^{2}+\frac{1}{4} \nabla_{\lambda} H_{\mu \alpha \beta} \nabla^{\lambda} H_{\nu}{ }^{\alpha \beta}+\frac{1}{12} \nabla_{\mu} H_{\alpha \beta \gamma} \nabla_{\nu} H^{\alpha \beta \gamma} \\
& \left.+\frac{1}{8} H_{\mu \alpha \lambda} H_{\nu \beta}{ }^{\lambda}\left(H^{2}\right)^{\alpha \beta}\right],
\end{aligned}
$$

where $H^{4}=H_{\mu \nu \lambda} H^{\nu \rho \kappa} H_{\rho \sigma}{ }^{\lambda} H^{\sigma \mu}{ }_{k}, H_{\mu \nu}^{2}=H_{\mu \rho \sigma} H_{\nu}^{\rho \sigma}$, and $H$ is the field strength of the $B$ field defined by $H_{\mu \nu \rho}=3 \partial_{[\mu} B_{\nu \rho]}$. The $f$ parameter indicates the $\mathrm{RS}$ dependence in $\beta$ functions. Especially, the schemes corresponding to $f=$ 1 and $f=-1$, called $R^{2}$ and Gauss-Bonnet schemes, have been pointed in Ref. [2]. Solutions of various RS $\beta$-function equations are different but still equivalent because of their belonging to various definitions of the physical metric, dilaton field, and $B$ field. The $\beta$ functions of the $B$ field in the mentioned RS are given by [2] 


$$
\begin{aligned}
\frac{1}{\alpha^{\prime}} \beta_{\mu \nu}^{B}(f=1)= & -\frac{1}{2} \nabla^{\mu} H_{\mu \nu \rho}+\frac{\alpha^{\prime}}{4}\left(2 R_{[\mu \gamma \alpha \beta} \nabla^{\gamma} H_{\nu]}^{\alpha \beta}\right. \\
& +\nabla_{\gamma} H_{\alpha \beta[\mu} H_{\nu] \rho}{ }^{\alpha} H^{\beta \gamma \rho}+2 \nabla_{\beta} H_{\alpha[\nu}^{2} H_{\mu]} \alpha \beta \\
& \left.+\frac{1}{2} H_{\alpha \beta}^{2} \nabla^{\alpha} H^{\beta}{ }_{\mu \nu}-\frac{1}{12} H_{\mu \nu}{ }^{\rho} \nabla_{\rho} H^{2}\right), \\
\frac{1}{\alpha^{\prime}} \beta_{\mu \nu}^{B}(f=-1)= & \hat{R}_{[\mu \nu]}+\frac{\alpha^{\prime}}{4}\left(2 \hat{R}^{\alpha \beta \gamma} \hat{[}_{[\nu} \hat{R}_{\mu] \alpha \beta \gamma}-\hat{R}_{[\nu}^{\beta \gamma \alpha} \hat{R}_{\mu] \alpha \beta \gamma}\right. \\
& \left.+\hat{R}_{\alpha[\mu \nu] \beta} H^{\alpha \beta}-\frac{1}{12} H_{\mu \nu}{ }^{\rho} \nabla_{\rho} H^{2}\right),
\end{aligned}
$$

where the $\hat{R}_{\mu \nu \sigma}^{\rho}$ is the Riemann tensor of the generalized connection with torsion $\hat{\Gamma}_{\mu \nu}^{\rho}=\Gamma_{\mu \nu}^{\rho}-\frac{1}{2} H_{\mu \nu}^{\rho}$ [36]. The averaged $\beta$ function of a dilaton, which can be written in terms of $\beta$ functions of the metric and dilaton field as $\tilde{\beta}^{\phi}=\beta^{\phi}-\frac{1}{4} \beta_{\mu \nu}^{g} g^{\mu \nu}$, is given by

$$
\begin{aligned}
\frac{1}{\alpha^{\prime}} \tilde{\beta}^{\phi}= & -R+\frac{1}{12} H^{2}+2 \nabla_{\mu} \nabla^{\mu} \phi+\left(\partial_{\mu} \phi\right)^{2}-\Lambda-\frac{\alpha^{\prime}}{4}\left(R_{\mu \nu \rho \lambda}^{2}\right. \\
& \left.-\frac{1}{2} R^{\alpha \beta \rho \sigma} H_{\alpha \beta \lambda} H_{\rho \sigma}{ }^{\lambda}+\frac{1}{24} H^{4}-\frac{1}{8}\left(H_{\mu \nu}{ }^{2}\right)^{2}\right)
\end{aligned}
$$

which can be obtained by variation of the following string effective action with respect to the dilaton field:

$$
\begin{aligned}
S= & \int d^{4} x \sqrt{G} e^{\phi}\left(R-\frac{1}{12} H^{2}+(\nabla \phi)^{2}+\Lambda+\frac{\alpha^{\prime}}{4}\left(R_{\mu \nu \rho \lambda}^{2}\right.\right. \\
& \left.\left.-\frac{1}{2} R^{\alpha \beta \rho \sigma} H_{\alpha \beta \lambda} H_{\rho \sigma}{ }^{\lambda}+\frac{1}{24} H^{4}-\frac{1}{8}\left(H_{\mu \nu}{ }^{2}\right)^{2}\right)\right) .
\end{aligned}
$$

The $\Lambda$ term is related to the central charge deficit of theory and in noncritical $D$-dimensional bosonic theory is given by $[16,17]$

$$
\Lambda=\frac{2(26-D)}{3 \alpha^{\prime}}
$$

The effective action (5) has been written in the string frame, and its variations with respect to the background fields give the $\beta$ functions. Also, there is another frame, namely, the Einstein frame, its metric, $\tilde{g}_{\mu \nu}$, of which is related to the string frame metric, $g_{\mu \nu}$, in four-dimensional space-time by

$$
\tilde{g}_{\mu \nu}=e^{\phi} g_{\mu \nu} .
$$

Actually, the $g_{\mu \nu}$ is the metric seen by the string and describes physics from the string viewpoint. However, it is not convenient to understand the gravitational phenomena due to the dilaton field-dependent coefficient of the Ricci scalar in (5). Transforming to the Einstein frame by performing the conformal transformation (7) eliminates the dilaton field-dependent factor. This frame is appropriate for comparison with the string $S$ matrix. Actually, computing the $\alpha^{\prime}$-corrected string effective action can be studied either in the $\sigma$ model and its $\beta$-functions approach or from the tree-level $S$ matrix. However, it is worth noting that to a given order $\alpha^{\prime}$ an intrinsic ambiguity remains in the string effective actions. Since the $S$ matrix is invariant under a set of field redefinitions of type [8]

$$
\begin{aligned}
\delta g_{\mu \nu}= & \alpha^{\prime}\left(b_{1} R_{\mu \nu}+b_{2} \partial_{\mu} \phi \partial_{\nu} \phi+b_{3} H_{\mu \nu}^{2}\right. \\
& \left.+g_{\mu \nu}\left(b_{4} R+b_{5}(\partial \phi)^{2}+b_{6} \nabla^{2} \phi+b_{7} H^{2}\right)\right), \\
\delta B_{\mu \nu}= & \alpha^{\prime}\left(b_{8} \nabla^{\lambda} H_{\lambda \mu \nu}+b_{9} H_{\mu \nu}{ }^{\lambda} \partial_{\lambda} \phi\right), \\
\delta \phi= & \alpha^{\prime}\left(b_{10} R+b_{11}(\partial \phi)^{2}+b_{12} \nabla^{2} \phi+b_{13} H^{2}\right),
\end{aligned}
$$

there is a field redefinition ambiguity and a class of physically equivalent effective actions parametrized by eight essential coefficients [37]. Choosing a particular set of field variables corresponds to a particular RS choice. Aimed at calculating a set of these coefficients, the GaussBonnet scheme has been used and gives the effective action for the bosonic string in four dimensions [2],

$$
\begin{aligned}
S= & \int d^{4} x \sqrt{G}\left(\tilde{R}-\frac{1}{12} e^{2 \phi} H^{2}-\frac{1}{2}(\tilde{\nabla} \phi)^{2}+\Lambda e^{-\phi}\right. \\
& +\frac{\alpha^{\prime} e^{\phi}}{4}\left[\tilde{R}_{\mu \nu \rho \lambda}^{2}-4 \tilde{R}_{\mu \nu}^{2}+\tilde{R}^{2}+e^{\phi}\left(\frac{1}{2} H_{\mu \nu}^{2} \tilde{\nabla}^{\mu} \phi \tilde{\nabla}^{\nu} \phi\right.\right. \\
& \left.-\frac{1}{2} \tilde{R}^{\alpha \beta \rho \sigma} H_{\alpha \beta \lambda} H_{\rho \sigma}{ }^{\lambda}+\frac{1}{2} H_{\mu \nu}^{2} \tilde{\nabla}^{\mu} \phi \tilde{\nabla}^{\nu} \phi-\frac{1}{12} H^{2}(\tilde{\nabla} \phi)^{2}\right) \\
& \left.\left.+e^{2 \phi}\left(\frac{1}{24} H^{4}+\frac{1}{8}\left(H_{\mu \nu}{ }^{2}\right)^{2}-\frac{5}{144}\left(H^{2}\right)^{2}\right)\right]\right),
\end{aligned}
$$

in which $\tilde{\nabla}$ indicates the covariant derivative with respect to $\tilde{g}$. The $\Lambda$, which is positive in $D=4$, appears in a way that reminds a negative cosmological constant in standard theory of gravity, up to a weight factor $e^{-\phi}$. Using the field redefinitions, the Gauss-Bonnet combination $\tilde{R}_{\mu \nu \rho \lambda}^{2}-$ $4 \tilde{R}_{\mu \nu}^{2}+\tilde{R}^{2}$ in the effective action can be replaced by the square of the Riemann tensor, and the price to be paid is the appearance of a dilaton-dependent $\alpha^{\prime}$ correction [2,6].

The equivalence of two-loop $\beta$ functions and equations of motion of the $\alpha^{\prime}$-corrected effective action can be established by using the field redefinitions and the lowest-order equations of motion [2]. Physical quantities are not affected by the field redefinitions [28], and appropriate use of them and the leading-order equations of motion allows us to transform between the RSs $[2,8]$. Hence, where the higherorder corrected field equations of effective actions are considered in the string theory context, the field redefinitions can be applied conveniently to reach the simplest effective action. In this sense, the Gauss-Bonnet effective action, which is free of ghosts and terms with higher than the second derivative in its field equations, usually holds attention. Considering the effective action (8), the variation over the Einstein frame metric metric, $\tilde{g}_{\mu \nu}$, gives

$$
\tilde{R}_{\mu \nu}-\frac{1}{2} \tilde{R} \tilde{g}_{\mu \nu}=T_{\mu \nu}^{(\mathrm{eff})}
$$


where the effective energy-momentum tensor is defined as

$$
T_{\mu \nu}^{(\mathrm{eff})}=T_{\mu \nu}^{(\phi)}+T_{\mu \nu}^{\left(B_{1}\right)}+T_{\mu \nu}^{(\mathrm{GB})}+T_{\mu \nu}^{\left(B_{2}\right)}
$$

in which the energy-momentum tensors of the dilaton field and $B$ field in the leading order are given by

$$
\begin{gathered}
T_{\mu \nu}^{(\phi)}=\frac{1}{2}\left(\tilde{\nabla}_{\mu} \phi \tilde{\nabla}_{\nu} \phi-\frac{1}{2} \tilde{g}_{\mu \nu}(\tilde{\nabla} \phi)^{2}+\Lambda e^{\phi} \tilde{g}_{\mu \nu}\right), \\
T_{\mu \nu}^{\left(B_{1}\right)}=\frac{e^{2 \phi}}{4}\left(H_{\mu \kappa \lambda} H_{\nu}^{\kappa \lambda}-\frac{1}{6} H^{2} \tilde{g}_{\mu \nu}\right),
\end{gathered}
$$

and in the $\alpha^{\prime}$ order, the Gauss-Bonnet term gives [38]

$$
\begin{aligned}
T_{\mu \nu}^{(\mathrm{GB})}= & \alpha^{\prime} e^{\phi}\left[-\frac{1}{2} \tilde{R}_{\mu \alpha \beta \gamma} \tilde{R}_{\nu}^{\alpha \beta \gamma}+\tilde{R}_{\mu \alpha \beta \nu} \tilde{R}^{\alpha \beta}+\tilde{R}_{\mu \alpha} \tilde{R}^{\nu \alpha}-\frac{1}{2} \tilde{R} \tilde{R}_{\mu \nu}+\frac{1}{8} \tilde{g}_{\mu \nu}\left(\tilde{R}_{\alpha \beta \rho \lambda}^{2}-4 \tilde{R}_{\alpha \beta}^{2}+\tilde{R}^{2}\right)\right. \\
& +\tilde{R}_{\mu \alpha \beta \nu}\left(\tilde{\nabla}^{\alpha} \tilde{\nabla}^{\beta} \phi+\tilde{\nabla}^{\alpha} \phi \tilde{\nabla}^{\beta} \phi\right)-2 \tilde{R}_{(\mu}^{\alpha}\left(\tilde{\nabla}_{\nu)} \tilde{\nabla}_{\alpha} \phi+\tilde{\nabla}_{\nu)} \phi \tilde{\nabla}_{\alpha} \phi\right)-\tilde{R}_{\mu \nu}\left((\tilde{\nabla} \phi)^{2}+\tilde{\nabla}^{2} \phi\right) \\
& \left.+\frac{1}{2} \tilde{R}\left(\tilde{\nabla}_{\mu} \phi \tilde{\nabla}_{\nu} \phi+\tilde{\nabla}_{\mu} \tilde{\nabla}_{\nu} \phi\right)+\left(\left(\tilde{\nabla}_{\rho} \tilde{\nabla}_{\sigma} \phi+\tilde{\nabla}_{\rho} \phi \tilde{\nabla}_{\sigma} \phi\right) \tilde{R}^{\rho \sigma}-\frac{1}{2}\left((\tilde{\nabla} \phi)^{2}+\tilde{\nabla}^{2} \phi\right) \tilde{R}\right) \tilde{g}_{\mu \nu}\right] .
\end{aligned}
$$

Also, we obtain the following energy-momentum tensor for the $B$ field-dependent $\alpha^{\prime}$-correction terms:

$$
\begin{aligned}
T_{\mu \nu}^{\left(B_{2}\right)}= & \frac{\alpha^{\prime} e^{3 \phi}}{8}\left[\left(\tilde{R}^{\alpha \beta \rho \sigma} H_{\mu \alpha \beta} H_{\nu \rho \sigma}+3 \tilde{R}^{\alpha \beta \sigma}{ }_{\mu} H_{\nu) \lambda \sigma} H_{\alpha \beta}^{\lambda}+e^{-3 \phi} \tilde{\nabla}^{\rho} \tilde{\nabla}^{\sigma}\left(e^{3 \phi} H_{\mu \rho \lambda} H_{\nu \sigma}{ }^{\lambda}\right)-\frac{1}{2}\left(H_{\alpha \mu \sigma} H_{\beta \nu}{ }^{\sigma} \tilde{\nabla}^{\alpha} \phi \tilde{\nabla}^{\beta} \phi\right.\right.\right. \\
& \left.\left.+H_{\beta(\mu}^{2} \tilde{\nabla}_{\nu)} \phi \tilde{\nabla}^{\beta} \phi\right)+\frac{1}{2}(\tilde{\nabla} \phi)^{2} H_{\mu \nu}^{2}+\frac{1}{12} H^{2} \tilde{\nabla}_{\mu} \phi \tilde{\nabla}_{\nu} \phi\right)-\frac{e^{2 \phi}}{2}\left(H_{\mu \nu}^{4}+H_{\mu \rho}^{2} H_{\nu}^{2 \rho}+2 H_{\alpha \beta}^{2} H_{\mu}^{\alpha \lambda} H_{\nu \lambda}{ }^{\beta}-\frac{5}{12} H^{2} H_{\mu \nu}^{2}\right) \\
& \left.+\frac{1}{2} \tilde{g}_{\mu \nu}\left(\left(-\tilde{R}^{\alpha \beta \rho \sigma} H_{\alpha \beta \gamma} H_{\rho \sigma}^{\gamma}-\tilde{\nabla}^{\rho} \phi \tilde{\nabla}^{\beta} \phi H_{\rho \beta}^{2}-\frac{1}{6} H^{2}(\tilde{\nabla} \phi)^{2}\right)+e^{2 \phi}\left(\frac{1}{12} H^{4}+\frac{1}{4}\left(H_{\alpha \beta}\right)^{2}-\frac{5}{72}\left(H^{2}\right)^{2}\right)\right)\right] .
\end{aligned}
$$

The $T_{\mu \nu}^{\mathrm{GB}}$ in (13) has been written in its most general case. Indeed, the Gauss-Bonnet term is a total derivative in four dimensions, and the terms without derivatives of the dilaton in $T_{\mu \nu}^{\mathrm{GB}}$ cancel each other and vanish automatically [38].

\section{NONCRITICAL $(\Lambda \neq 0)$ ANISOTROPIC BIANCHI TYPE I TWO-LOOP STRING COSMOLOGY SOLUTIONS}

In this section, we are going to solve the two-loop $\beta$ functions in the presence of central charge deficit $\Lambda$ on Bianchi type I space-time with a similar method by which we have calculated the solutions of two-loop $\beta$ functions on homogeneous space-times in Ref. [20]. The solutions give the string frame metric $g_{\mu \nu}$, dilaton field, and $B$ field, where the corresponding Einstein frame solutions can be obtained using the conformal transformation (7). Maintaining the provided convention by $\beta$-function solutions, the field redefinitions of Ref. [8] will not be applied.

Considering the anisotropic Bianchi type I metric as a string frame metric,

$d s^{2}=g_{\mu \nu} d x^{\mu} d x^{\nu}=-g_{00}(t) d t^{2}+\sum_{i=1}^{3} a_{i}^{2}(t)\left(d x^{i}\right)^{2}$,

where $a_{i}$ are the string frame scale factors, we have

$$
\begin{aligned}
\Gamma_{i j}^{t} & =g^{00} a_{i}(t)^{2} H_{i} \delta_{j}^{i}, \\
\Gamma_{j t}^{i} & =H_{i} \delta_{j}^{i}, \\
\Gamma_{t t}^{t} & =H_{0}, \\
R_{i j i j} & =g^{00}\left(a_{i} a_{j}\right)^{2} H_{i} H_{j}, \\
R_{i t i t} & =-a_{i}^{2}\left(\dot{H}_{i}-H_{i} H_{0}+H_{i}^{2}\right), \\
R_{t t} & =-\sum\left(\dot{H}_{i}+H_{i}^{2}-H_{0} H_{i}\right), \\
R_{i i} & =\dot{H}_{i}+H_{i} \sum H_{k}-H_{0} H_{i}, \\
R & =g^{00}\left(\sum\left(2 \dot{H}_{i}+H_{i}^{2}-2 H_{0} H_{i}\right)+\left(\sum H_{i}\right)^{2}\right) .
\end{aligned}
$$

The dot symbol stands for derivation with respect to $t$, and the $H_{i}$ are the Hubble coefficients of the string frame defined by $H_{i}=\frac{d}{d t}\left(\ln a_{i}\right)$ and $H_{0}=\frac{1}{2} \frac{d}{d t}\left(\ln g_{00}\right)$.

The solutions of the $\beta$-function equations will be investigated in two cases of the absence and presence of the $H$ field. Since the considered metric is spatially homogeneous, the dilaton field can be only a function of time.

\section{A. Solutions with vanishing $\boldsymbol{H}$ field}

Without the contribution of the $H$ field, the $(i, i)$ and time-time components of the metric $\beta$ function (1) and the $\beta$ function of the dilaton (4) with using relations (16) reduce the coupled differential equations 


$$
\begin{gathered}
\dot{H}_{i}+H_{i} \sum H_{k}+H_{i} \dot{\phi}-H_{i} H_{0}+\alpha^{\prime} K_{i} g^{00}=0 \\
\ddot{\phi}+\sum\left(\dot{H}_{i}+H_{i}^{2}-H_{0}\left(\dot{\phi}+\dot{H}_{i}\right)\right)+\alpha^{\prime} K_{0} g^{00}=0 \\
-2 \ddot{\phi}-\dot{\phi}^{2}-\sum\left(2 \dot{\phi} H_{i}+H_{i}^{2}+2 \dot{H}_{i}\right)-\left(\sum H_{i}\right)^{2}-\Lambda g_{00} \\
-2\left(\dot{\phi}+\sum H_{i}\right) H_{0}-\alpha^{\prime} K_{\phi} g^{00}=0
\end{gathered}
$$

where the auxiliary $K$ functions have been introduced for the shorthanded writing of equations and are given as follows:

$$
\begin{aligned}
K_{i}= & \dot{H}_{i}^{2}+2\left(H_{i}-H_{0}\right) H_{i} \dot{H}_{i}+H_{i}^{2}\left(\sum H_{k}^{2}-H_{0}^{2}-H_{0} H_{i}\right), \\
K_{0}= & \sum\left(\dot{H}_{i}^{2}+2 H_{i}\left(H_{i}-H_{0}\right) \dot{H}_{i}+H_{i}^{4}+H_{0}^{2} H_{i}^{2}-H_{0} H_{i}^{3}\right), \\
K_{\phi}= & \sum\left(\dot{H}_{i}^{2}+2 H_{i}\left(H_{i}-H_{0}\right) \dot{H}_{i}+H_{i}^{4}\right. \\
& \left.+H_{0}^{2} H_{i}^{2}-H_{0} H_{i}^{3}\right)+\sum_{i<j} H_{i}^{2} H_{j}^{2} .
\end{aligned}
$$

Now, adding Eqs. (18) and (19) with sum-over $i$ in Eq. (17) leads to the following equation:

$$
\begin{gathered}
-\ddot{\phi}-\dot{\phi}\left(\dot{\phi}+\sum H_{i}\right)+\dot{\phi} H_{0}-\Lambda g_{00} \\
+\alpha^{\prime}\left(K_{\phi}+K_{0}+\sum K_{i}\right) g^{00}=0 .
\end{gathered}
$$

Also, subtracting the summed-over $i$ of (17) from the sum of Eqs. (18) and (21) gives the initial value equation as follows:

$$
\begin{aligned}
& \dot{\phi}\left(\dot{\phi}+2 \sum H_{i}\right)+\left(\sum H_{i}\right)^{2}-\sum H_{i}^{2}+\Lambda g_{00} \\
& \quad-\alpha^{\prime}\left(K_{\phi}+2 K_{0}\right) g^{00}=0 .
\end{aligned}
$$

We are going to solve the set of equations of (17) and (21) subject to the initial value equation (22) along with implementing the perturbative series expansion on the background fields up to the first order of $\alpha^{\prime}$,

$$
\begin{gathered}
\phi=\phi_{0}+\alpha^{\prime} \phi_{1}, \\
a_{i}^{2}=a_{i 0}^{2}\left(1+2 \alpha^{\prime} \xi_{i}\right), \\
g_{00}=1+2 \alpha^{\prime} \xi_{4},
\end{gathered}
$$

and applying a time redefinition that introduces the new time coordinate $\tau$ as [11]

$$
d \tau=a^{-3} \mathrm{e}^{-\phi} d t
$$

where $a^{3}=a_{1} a_{2} a_{3}$. Accordingly, Eqs. (17) and (21) recast the equations in the zeroth order of $\alpha^{\prime}$,

$$
\begin{gathered}
\left(\ln a_{i 0}\right)^{\prime \prime}=0, \\
\phi_{0}^{\prime \prime}+\Lambda a_{0}^{6} \mathrm{e}^{2 \phi_{0}}=0,
\end{gathered}
$$

where $a_{0}^{3}=a_{10} a_{20} a_{30}$. Also, in the first order of $\alpha^{\prime}$, we get

$$
\begin{gathered}
\xi_{i}^{\prime \prime}-\left(\ln a_{i 0}\right)^{\prime} \xi_{4}^{\prime}+\hat{K}_{i}=0, \\
\phi_{1}^{\prime \prime}+2 \Lambda a_{0}^{6} \mathrm{e}^{2 \phi_{0}}\left(\phi_{1}+\sum \xi_{i}+\xi_{4}\right)-\phi_{0}^{\prime} \xi_{4}^{\prime} \\
-\hat{K}_{\phi}-\sum \hat{K}_{i}-\hat{K}_{0}=0,
\end{gathered}
$$

where the prime stands for derivations with respect to $\tau$ and the $\hat{K}$ terms are the rewritten versions of $K$ terms in the new time coordinate, multiplied with a $a^{6} \mathrm{e}^{2 \phi}$ factor. Also, the initial value equation (22) reads [39]

$$
\begin{aligned}
& \frac{1}{2}\left[\sum_{i<j}\left(\ln a_{i 0}^{2} \mathrm{e}^{\phi_{0}}\right)^{\prime}\left(\ln a_{j 0}^{2} \mathrm{e}^{\phi_{0}}\right)^{\prime}-\phi_{0}^{\prime 2}+2 \Lambda a_{0}^{6} \mathrm{e}^{2 \phi_{0}}\right] \\
& \quad+\alpha^{\prime}\left[\left(\phi_{0}^{\prime}+\sum \ln a_{i 0}^{\prime}\right)\left(\phi_{1}^{\prime}+\sum \xi_{j}^{\prime}\right)-2 \sum \ln a_{i 0}^{\prime} \xi_{i}^{\prime}\right. \\
& \left.\quad+2 \Lambda a_{0}^{6} \mathrm{e}^{2 \phi_{0}}\left(\phi_{1}+\sum \xi_{i}+\xi_{4}\right)+\hat{K}_{\phi}+2 \hat{K}_{0}\right]=0 .
\end{aligned}
$$

The solution of (27) and (28) gives the zeroth order of scale factors and dilaton field as

$$
\begin{gathered}
a_{i 0}=L_{i} \mathrm{e}^{q_{i} \tau}, \\
\phi_{0}=-\sum q_{i} \tau-\ln \left(\frac{\sqrt{\Lambda} L_{1} L_{2} L_{3}}{n} \cosh (n \tau)\right),
\end{gathered}
$$

where $L_{i}, q_{i}$, and $n$ are integrating constants. Accordingly, the leading-order string frame Ricci scalar and kinetic of the dilaton field are given by

$R=\frac{\Lambda}{n^{2}} \cosh ^{2}(n \tau)\left(\left(\sum q_{i}\right)^{2}+\sum q_{i}\left(q_{i}+2 n \tanh (n \tau)\right)\right)$,

$$
\begin{aligned}
\dot{\phi}_{0}^{2}= & a_{0}^{-6} e^{-2 \phi_{0}} \phi^{\prime 2}=\frac{\Lambda}{n^{2}}\left(\left(n^{2}+\left(\sum q_{i}\right)^{2}\right) \cosh ^{2}(n \tau)\right. \\
& \left.+2 n \sum q_{i} \cosh (n \tau) \sinh (n \tau)-n^{2}\right),
\end{aligned}
$$

which are growing functions of time. The dependence of $R$ and $\dot{\phi}_{0}^{2}$ on $\Lambda$, which implies that the curvature and dilaton field kinetic are comparable with inverse of $\alpha^{\prime}$ [40], demonstrates the necessity of including the higher orders of $\alpha^{\prime}$ corrections. In this work, we focus on studying the effects of first order of $\alpha^{\prime}$ corrections. 
Demanding only the first-order $\alpha^{\prime}$ corrections in the solutions of the metric, dilaton field, and $H$ field, only the zeroth-order $\alpha^{\prime}$ terms of $\hat{K}$ will be considered. Hence, substituting (32) and (33) into the rewritten versions of (20) in the $\tau$ coordinate gives the explicit forms of $\hat{K}$. Now, the solutions of (29) and (30) give the general forms of $\alpha^{\prime}$ corrections of the scale factor and dilaton field as

$$
\begin{aligned}
& \xi_{i}=-\iint \hat{K}_{i} d \tau d \tau+q_{i} \int \xi_{4} d \tau+l_{i} \tau+r_{i}, \\
\phi_{1}= & Q_{1} \tanh (n \tau)+Q_{2}(n \tau \tanh (n \tau)-1)+\varphi_{0} \\
+ & \frac{1}{n}\left(\tanh (n \tau) \int(n \tau \tanh (n \tau)-1) g(\tau) d \tau\right. \\
- & \left.(n \tau \tanh (n \tau)-1) \int \tanh (n \tau) g(\tau) d \tau\right) \\
- & \left(n \tanh (n \tau)+\sum q_{i}\right) \int \xi_{4} d \tau,
\end{aligned}
$$

in which $g(\tau)$ is given by

$g(\tau)=\sum\left(\frac{-2 n^{2}}{\cosh ^{2}(n \tau)} \iint \hat{K}_{i} d \tau d \tau+\hat{K}_{i}\right)-\hat{K}_{\phi}-\hat{K}_{0}$

and $l_{i}, r_{i}, \varphi_{0}, Q_{1}$, and $Q_{2}$ are constants of integration. After some calculations with using Taylor series expansions up to the first order of $\alpha^{\prime}$, it turns out that these constants have the following roles:

(i) $l_{i}$ corresponds to an infinitesimal change in $q_{i}, q_{i} \rightarrow q_{i}+\alpha^{\prime} l_{i}$.

(ii) $r_{i}$ is a proper scaling in the $x_{i}$ direction.

(iii) $Q_{1}$ describes an infinitesimal time displacement, $\tau \rightarrow \tau-\alpha^{\prime} Q_{1}$.

(iv) $Q_{2}$ corresponds to an infinitesimal change in $n, n \rightarrow n\left(1-\alpha^{\prime} Q_{2}\right)$.

(v) $\varphi_{0}$ describes a constant shift in the dilaton.

Substituting these solutions into the initial value equation (31) gives

$$
\begin{aligned}
\left(n^{2}\right. & \left.-\sum q_{i}^{2}\right)\left(1+2 \alpha^{\prime} \xi_{4}\right) \\
= & 2 \alpha^{\prime}\left[n^{2} Q_{2}+\frac{1}{2} \hat{K}_{\phi}+\hat{K}_{0}\right. \\
& +\sum\left(\frac{n^{2}}{\cosh ^{2}(n \tau)} \iint \hat{K}_{i} d \tau d \tau-(n \tanh (n \tau)\right. \\
& \left.\left.\left.+q_{i}\right) \int \hat{K}_{i} d \tau\right)+n \int \tanh (n \tau) g(\tau) d \tau\right] .
\end{aligned}
$$

Actually, the $n^{2}-\sum q_{i}^{2}$ term is the initial condition on the constants, which appears in the one-loop $\beta$-function solutions. However, here, we are not allowed to set it to zero because the right side of this equation does not vanish. A comparison between the two sides of this equation proposes the initial condition on arbitrary constants,

$$
n^{2}\left(1-2 \alpha^{\prime} Q_{2}\right)-\sum q_{i}^{2}=0
$$

and the remaining terms on the right side of (39) fix the correction of the lapse function, $\xi_{4}$. In the $\alpha^{\prime} \rightarrow 0$ limit, the zeroth-order initial condition can be recovered from (40). Appearing the $\alpha^{\prime} Q_{2}$ term in the constraint would not be disappointing because, as we have mentioned before, $Q_{2}$ can be related to an infinitesimal change in $n$ that acts as $n^{2} \rightarrow n^{2}\left(1-2 \alpha^{\prime} Q_{2}\right)$ up to first order of $\alpha^{\prime}$. Noting the relation between $\beta$-function equations and Einstein equations, this initial condition may be regarded as a Hamiltonian constraint that has been corrected in first order $\alpha^{\prime}$.

The explicit forms of $\alpha^{\prime}$ corrections of the metric and dilaton after calculating the integrals in (36), (37), and (39) are presented in Appendix A.

\section{B. Solution with nonvanishing $\boldsymbol{H}$ field of a spatially homogeneous (time-dependent) $B$ field}

As mentioned before, the forms of the $H$ field can be classified based on the orientation of its dual, $H^{*}$, with respect to the 3D hypersurface of homogeneity $\Sigma^{3}$ sections. Accordingly, the three classes of $\rightarrow, \uparrow$, and $\nearrow$ denoting the spatial, time, and time-spatial orientations of $H^{*}$ have been introduced, respectively, by [12]

$H^{*}=H_{i}^{*}(t) d x^{i}, \quad H^{*}=H_{0}^{*} d t, \quad H^{*}=H_{0}^{*}(t) d t+H_{i}^{*}(t) d x^{i}$.

With $\Lambda=0$, the solution of one-loop $\beta$-function equations has been investigated on Bianchi-type models for $\uparrow$, $\rightarrow$, and $\nearrow$ classes in Refs. [10-12], respectively.

With a nonvanishing $\Lambda$, we have found no explicit solution for the leading order of $\beta$-function equations in the $\uparrow$ and $\nearrow$ classes. Therefore, we keep going with the $\rightarrow$ class with the metric (15) in such a way that, considering a $B$ field that is a function of time, the $H_{0 i j}$ components of the $H$ field are allowed to be nonzero [41]. On the other hand, the leading-order $\beta$-function equations with metric (15) make the off-diagonal components of $H_{\mu \nu}^{2}$ vanish. This means that only one of the $H_{0 i j}$ may be nonzero. Here, there is no preferred direction, and we consider the following 3-form of field strength $H$ for simplicity [10]:

$$
H=\frac{1}{3} A(t)\left(a_{1} a_{2}\right)^{2}\left(d t \wedge d x^{1} \wedge d x^{2}\right)
$$

Then, the $\beta$-function equations (1)-(4), using (16), recast the forms 


$$
\begin{gathered}
\dot{H}_{k}+H_{j}\left(\sum H_{i}+\dot{\phi}-H_{0}\right)+\frac{1}{2} A^{2}\left(a_{1} a_{2}\right)^{2}+\alpha^{\prime}\left(K_{j}+V_{j}\right) g^{00}=0, \quad j=1,2 \\
\dot{H}_{3}+H_{3}\left(\sum H_{k}+\dot{\phi}-H_{0}\right)+\alpha^{\prime}\left(K_{3}+V_{3}\right) g^{00}=0 \\
\sum\left(\dot{H}_{i}+H_{i}^{2}\right)+\ddot{\phi}-\frac{1}{2} A^{2}\left(a_{1} a_{2}\right)^{2}-H_{0}\left(\dot{\phi}+\sum \dot{H}_{i}\right)-\alpha^{\prime}\left(K_{0}+V_{0}\right) g^{00}=0 \\
\dot{A}+A\left(\dot{\phi}+\sum H_{i}-H_{0}\right)+\alpha^{\prime} g^{00} V_{B}=0 \\
-2 \ddot{\phi}-\dot{\phi}^{2}-\sum\left(2 \dot{\phi} H_{i}+H_{i}^{2}+2 \dot{H}_{i}\right)-\left(\sum H_{i}\right)^{2}-\Lambda g_{00}-\frac{1}{2} A^{2}\left(a_{1} a_{2}\right)^{2}-2\left(\dot{\phi}+\sum H_{i}\right) H_{0}-\alpha^{\prime}\left(K_{\phi}+V_{\phi}\right) g^{00}=0
\end{gathered}
$$

where the $K$ terms are the same as given in (20) and the $V$ terms, which stand for the $H$ field-dependent terms, are given as follows:

$$
\begin{aligned}
& V_{1}= {\left[\frac { 1 } { 2 } \left(\left(3 H_{2}^{2}+\left(-H_{0}+4 H_{1}\right) H_{2}+2 H_{0}^{2}+2 H_{1}^{2}+2 H_{3}^{2}+\dot{H}_{2}\right) f+2 H_{2}^{2}+\left(-H_{0}+4 H_{1}\right) H_{2}+H_{0}^{2}-2 H_{0} H_{1}\right.\right.} \\
&+\left.\left.3 H_{1}^{2}+H_{3}^{2}+\dot{H}_{2}\right) A^{2}-\left(\left(H_{0}-H_{1}-H_{2}\right) f+\frac{1}{2} H_{0}-\frac{1}{2} H_{2}\right) \dot{A} A+\frac{1}{2}(1+f) \dot{A}^{2}\right]\left(a_{1} a_{2}\right)^{2}+\frac{3}{16}\left(a_{1} a_{2}\right)^{4} A^{4}, \\
& V_{2}=V_{1}(1 \leftrightarrow 2), \\
& V_{3}=-\frac{1}{2}\left[A \dot{H}_{3} f+H_{3} A\left(\left(-H_{0}+H_{1}+H_{2}-H_{3}\right) f-H_{0}+H_{1}+H_{2}-2 H_{3}\right)+H_{3} \dot{A}\right] A\left(a_{1} a_{2}\right)^{2}, \\
& V_{0}=\frac{1}{2}\left[\left(\left(2 H_{0}^{2}-\left(H_{1}+H_{2}\right) H_{0}+3 H_{1}^{2}+5 H_{1} H_{2}+3 H_{2}^{2}+\dot{H}_{2}+\dot{H}_{1}\right) f-H_{0}^{2}+2\left(H_{1}+H_{2}\right) H_{0}+3 H_{1}^{2}+H_{1} H_{2}+3 H_{2}^{2}\right.\right. \\
&\left.\left.+2\left(\dot{H}_{2}+\dot{H}_{1}\right)+\dot{H}_{0}\right) A^{2}-\dot{A}\left(2\left(H_{0}-H_{1}-H_{2}\right) f-3 H_{0}+H_{1}+H_{2}\right) A+\dot{A}^{2} f-A \ddot{A}\right]\left(a_{1} a_{2}\right)^{2}+\frac{3}{8}\left(a_{1} a_{2}\right)^{4} A^{4}, \\
& V_{B}^{(f=1)}=\frac{1}{2}\left[\left(H_{1}^{2}+H_{2}^{2}-H_{0}\left(H_{1}+H_{2}\right)+\dot{H}_{1}+\dot{H}_{2}\right) \dot{A}+A\left(\left(H_{1}+H_{2}-H_{0}\right)\left(\dot{H}_{1}+\dot{H}_{2}\right)+\left(H_{1}+H_{2}\right) \sum H_{i}^{2}\right.\right. \\
&-\left.\left.H_{0}\left(2\left(H_{1}^{2}+H_{2}^{2}+H_{1} H_{2}\right)-H_{0}\left(H_{1}+H_{2}\right)\right)\right)-A^{2}\left(15 \dot{A}+A\left(13\left(H_{1}+H_{2}\right)-16 H_{0}\right)\right)\left(a_{1} a_{2}\right)^{2}\right] \\
& V_{B}^{(f=-1)}= \frac{1}{2}\left[\left(H_{1}^{2}+H_{2}^{2}-H_{0}\left(H_{1}+H_{2}\right)+\dot{H}_{1}+\dot{H}_{2}\right) \dot{A}+A\left(\left(H_{1}+H_{2}-H_{0}\right)\left(\dot{H}_{1}+\dot{H}_{2}\right)+\left(H_{1}+H_{2}\right) \sum H_{i}^{2}\right.\right. \\
&\left.\left.-2 H_{0}\left(H_{1}^{2}+H_{2}^{2}+H_{1} H_{2}-H_{0}\left(H_{1}+H_{2}\right) / 2\right)\right)-11 A^{2}\left(\dot{A}+A\left(H_{1}+H_{2}-H_{0}\right)\right)\left(a_{1} a_{2}\right)^{2}\right] \\
& V_{\phi}=-\frac{1}{2}\left[\dot{H}_{1}+\dot{H}_{2}+H_{1}^{2}+\left(H_{2}-H_{0}\right) H_{1}+H_{2}^{2}-H_{0} H_{2}\right] A^{2}\left(a_{1} a_{2}\right)^{2}+\frac{1}{4}\left(a_{1} a_{2}\right)^{4} A^{4} .
\end{aligned}
$$

Similar to what we have done to obtain Eq. (21), adding (42)-(44) to (46) gives the following equation:

$$
-\ddot{\phi}-\dot{\phi}\left(\dot{\phi}+\sum H_{i}\right)+\dot{\phi} H_{0}-\Lambda g_{00}+A^{2}\left(a_{1} a_{2}\right)^{2}+\alpha^{\prime}\left(K_{\phi}+V_{\phi}+K_{0}+V_{0}+\sum\left(K_{i}+V_{i}\right)\right) g^{00}=0 .
$$

Furthermore, adding (54) to (44) and subtracting (42) and (43) from it give the initial value equation as follows:

$$
\dot{\phi}\left(\dot{\phi}+2 \sum H_{i}\right)+\left(\sum H_{i}\right)^{2}-\sum H_{i}^{2}+\Lambda g_{00}-\frac{1}{2} A^{2}\left(a_{1} a_{2}\right)^{2}-\alpha^{\prime}\left(K_{\phi}+V_{\phi}+2\left(K_{0}+V_{0}\right)\right) g^{00}=0 .
$$

Now, we are going to solve Eqs. (42), (43), (45), and (54) subject to the initial value equation (55). Again, the equations will be rewritten in the new time coordinate (26) with applying the given series expansion in $\alpha^{\prime}(23)-(25)$. Also, we will conveniently set 


$$
A(t)=\dot{\eta}=\eta^{\prime} a^{-3} \mathrm{e}^{-\phi}
$$

and take the $\alpha^{\prime}$ expansion of $\eta$ as

$$
\eta=\eta_{0}+\alpha^{\prime} \eta_{1}
$$

Then, in the $\tau$ coordinate, Eqs. (42), (43), (45), and (54) lead to the following equations in the zeroth order of $\alpha^{\prime}$ :

$$
\begin{gathered}
\left(\ln a_{j 0}\right)^{\prime \prime}+\frac{1}{2} \eta_{0}^{\prime 2}\left(a_{10} a_{20}\right)^{2}=0, \quad j=1,2, \\
\left(\ln a_{30}\right)^{\prime \prime}=0 \\
\eta_{0}^{\prime \prime}=0 \\
\phi_{0}^{\prime \prime}-\eta_{0}^{\prime 2}\left(a_{10} a_{20}\right)^{2}+\Lambda a_{0}^{6} \mathrm{e}^{2 \phi_{0}}=0 .
\end{gathered}
$$

The solutions of above equations are found as

$a_{10}=\frac{\sqrt{n} \mathrm{e}^{-q \tau}}{\sqrt{b L_{2} \cosh (n \tau)}}, \quad a_{20}=L_{2} \mathrm{e}^{2 q \tau} a_{10}, \quad a_{30}=L_{3} \mathrm{e}^{p \tau}$,

$$
\eta_{0}=b \tau,
$$

$\phi_{0}=-p \tau+\ln \cosh (n \tau)-\ln \left(\frac{n \Lambda L_{3}}{m b} \cosh (m \tau)\right)$,

where $q, L_{2}, L_{3}, b, p, m$, and $n$ are constant. Obviously, the $H$ field in this class brings about an inevitable anisotropy in the solutions. Compared to the leading-order solutions in the absence of $\Lambda$ given in Ref. [10], only the dilaton field has been modified by the third term in (64). Based on these solutions, the leading-order string frame Rici scalar and kinetic terms of the dilaton field and $B$ field, with $m=n$, for example, are given by

$$
\begin{gathered}
R=\frac{\Lambda}{2 n^{2}}\left(\left(-n^{2}+4 p^{2}+4 q^{2}\right) \cosh ^{2}(n \tau)-3 n^{2}\right), \\
\dot{\phi}_{0}^{2}=a_{0}^{-6} e^{-2 \phi_{0}} \phi^{\prime 2}=\frac{p^{2} \Lambda}{n^{2}} \cosh ^{2}(n \tau), \\
H_{\mu \nu \rho} H^{\mu \nu \rho}=6 \eta^{\prime 2} a_{03}^{-2} e^{-2 \phi_{0}}=6 \Lambda .
\end{gathered}
$$

Evidently, the string frame Ricci scalar is increasing and starts from the high curvature limit $R \alpha^{\prime} \gtrsim 1$. Also, the kinetic terms are comparable with the inverse of $\alpha^{\prime}$. Note that the $R$ and $\dot{\phi}_{0}^{2}$ keep growing and may dominate the dynamical effect of the $H$ field at late $\tau$. The high curvature and kinetic terms point out the necessity of considering the $\alpha^{\prime}$ corrections. We include the first-order $\alpha^{\prime}$ corrections, noting that the solutions are valid as long as the string coupling is weak.
In the first order of $\alpha^{\prime}$ by employing the zeroth-order equations (58)-(61), Eqs. (42), (43), (45), and (54) read

$$
\begin{gathered}
\xi_{j}^{\prime \prime}-\left(\ln a_{j 0}\right)^{\prime} \xi_{4}^{\prime}+\left(\eta_{0}^{\prime 2}\left(\xi_{1}+\xi_{2}\right)+\eta_{0} \eta_{1}\right)\left(a_{10} a_{20}\right)^{2} \\
+\hat{K}_{j}+\hat{V}_{j}=0, \quad j=1,2, \\
\xi_{3}^{\prime \prime}-\left(\ln a_{30}\right)^{\prime} \xi_{4}^{\prime}+\hat{K}_{3}+\hat{V}_{3}=0, \\
\eta_{1}^{\prime \prime}-\eta_{0}^{\prime} \xi_{4}^{\prime}+\hat{V}_{B}=0, \\
\phi_{1}^{\prime \prime}+2 \Lambda a_{0}^{6} \mathrm{e}^{2 \phi_{0}}\left(\phi_{1}+\sum \xi_{i}+\xi_{4}\right) \\
+2\left(\eta_{0}^{\prime 2}\left(\xi_{1}+\xi_{2}\right)+\eta_{0} \eta_{1}\right)\left(a_{10} a_{20}\right)^{2}-\phi_{0}^{\prime} \xi_{4}^{\prime}+\rho=0,
\end{gathered}
$$

where the $\hat{K}$ and $\hat{V}$ terms are the corresponding terms of (20) and (48)-(53) rewritten in the new $\tau$ coordinate, multiplied with a $a^{6} e^{2 \phi}$ factor. Also, the $\rho$ term in (71) has been defined as

$$
\rho=-\left(\hat{K}_{\phi}+\hat{V}_{\phi}+\sum\left(\hat{K}_{i}+\hat{V}_{i}\right)+\hat{K}_{0}+\hat{V}_{0}\right) .
$$

In the same way, the initial value equation (55) is recast into the following form:

$$
\begin{aligned}
& \frac{1}{2}\left[\sum_{i<j}\left(\ln a_{i 0}^{2} \mathrm{e}^{\phi_{0}}\right)^{\prime}\left(\ln a_{j 0}^{2} \mathrm{e}^{\phi_{0}}\right)^{\prime}-\phi_{0}^{\prime 2}+2 \Lambda a_{0}^{6} \mathrm{e}^{2 \phi_{0}}\right. \\
& \left.\quad-\frac{1}{2} \eta_{0}^{\prime 2}\left(a_{10} a_{20}\right)^{2}\right]+\alpha^{\prime}\left[\left(\phi_{0}^{\prime}+\sum \ln a_{i 0}^{\prime}\right)\left(\phi_{1}^{\prime}+\sum \xi_{j}^{\prime}\right)\right. \\
& \quad-2 \sum \ln a_{i 0}^{\prime} \xi_{i}^{\prime}+2 \Lambda a_{0}^{6} \mathrm{e}^{2 \phi_{0}}\left(\phi_{1}+\sum \xi_{i}+\xi_{4}\right) \\
& \quad-\left(\eta_{0}^{\prime 2}\left(\xi_{1}+\xi_{2}\right)+\eta_{0} \eta_{1}\right)\left(a_{10} a_{20}\right)^{2} \\
& \left.\quad+\hat{K}_{\phi}+\hat{V}_{\phi}+2\left(\hat{K}_{0}+\hat{V}_{0}\right)\right]=0 .
\end{aligned}
$$

Again, because we are interested in the first-order $\alpha^{\prime}$ corrections in the solutions of the metric, dilaton field, and $H$ field, only the zeroth-order $\alpha^{\prime}$ terms of $\hat{K}$ and $\hat{V}$, which depend on $a_{i 0}, \phi_{0}$ and $\eta_{0}$, will be considered. Hence, substituting (62)-(64) into the rewritten versions of (20) and (48)-(53) in the $\tau$ coordinate gives the explicit forms of $\hat{K}$ and $\hat{V}$. Then, solving Eqs. (68)-(71) gives the general forms of $\alpha^{\prime}$ corrections of the scale factors $\xi_{i}$, lapse function $\xi_{4}, H$ field $\eta_{1}$, and dilaton field $\phi_{1}$ as 


$$
\begin{aligned}
\xi_{1}= & c_{1} \tanh (n \tau)+c_{2}(n \tau \tanh (n \tau)-1)+r_{1} \\
& +\left(\frac{n}{2} \tanh (n \tau)-q\right) \int \xi_{4} d \tau \\
& +\frac{1}{n}\left[\tanh (n \tau) \int(n \tau \tanh (n \tau)-1) g_{1}(\tau) d \tau\right. \\
& \left.-(n \tau \tanh (n \tau)-1) \int \tanh (n \tau) g_{1}(\tau) d \tau\right], \\
\xi_{2}= & \xi_{1}-\iint\left(\hat{K}_{2}+\hat{V}_{2}-\hat{K}_{1}-\hat{V}_{1}\right) d \tau d \tau+2 q \int \xi_{4} d \tau, \\
& \xi_{3}=-\iint\left(\hat{K}_{3}+\hat{V}_{3}\right) d \tau d \tau+p \int \xi_{4} d \tau+r_{3}, \\
\phi_{1}= & \frac{1}{m}\left[\tanh (m \tau) \int(m \tau \tanh (m \tau)-1) g_{\phi}(\tau) d \tau\right. \\
& \left.-(m \tau \tanh (m \tau)-1) \int \tanh (m \tau) g_{\phi}(\tau) d \tau\right]+\varphi_{0},
\end{aligned}
$$

where $g_{1}$ and $g_{\phi}$ are given by

$$
\begin{gathered}
g_{1}(\tau)=-\frac{2 n^{2}}{\cosh ^{2}(n \tau)}\left(\iint\left(\hat{K}_{2}+\hat{V}_{2}-\hat{K}_{1}-\hat{V}_{1}\right) d \tau d \tau\right. \\
\left.-q_{0}^{-1} \int \hat{V}_{B} d \tau\right)+\hat{K}_{1}+\hat{V}_{1}, \\
g_{\phi}(\tau)=2 \Lambda a_{0}^{6} \mathrm{e}^{2 \phi_{0}}\left(\sum \xi_{i}+\xi_{4}\right)-\phi_{0}^{\prime} \xi_{4}^{\prime}+\rho \\
+2\left(\eta_{0}^{\prime 2}\left(\xi_{1}+\xi_{2}\right)+\eta_{0} \eta_{1}\right)\left(a_{10} a_{20}\right)^{2}
\end{gathered}
$$

and $c_{1}, c_{2}, r_{i}$, and $\varphi_{0}$ are integrating constants. A closer look with using Taylor series expansion reveals that up to first order of $\alpha^{\prime}$ :

(i) $c_{1}$ corresponds to an infinitesimal time displacement $\tau \rightarrow \tau-2 \alpha^{\prime} c_{1}$.

(ii) $c_{2}$ acts as an infinitesimal change in $n$, $n \rightarrow n\left(1-2 \alpha^{\prime} c_{2}\right)$.

(iii) $\varphi_{0}$ is an infinitesimal shift in the dilaton.

(iv) $r_{i}$ is a proper scaling in the $x_{i}$ direction.

For calculating the integrals of (78), it is convenient to set $m=n$, where, as given in Appendix $\mathrm{B}$, the $\phi_{1}$ and $g_{\phi}$ take the forms of (B1) and (B2). Then, similar to what has been done in the previous subsection, substituting the above solutions into the initial value equation (73) gives

$$
\begin{aligned}
& \frac{1}{2}\left(n^{2}-2 p^{2}-4 q^{2}\right)\left(1+2 \alpha^{\prime} \xi_{4}\right)=\alpha^{\prime}\left[2 n^{2} c_{2}\right. \\
& -2 n \tanh (n \tau) \int\left(\hat{K}_{3}+\hat{V}_{3}\right) d \tau+\hat{K}_{\phi}+\hat{V}_{\phi}+2 \hat{K}_{0}+2 \hat{V}_{0} \\
& +(n \tanh (n \tau)+2 q) \int\left(\hat{K}_{2}+\hat{V}_{2}-\hat{K}_{1}-\hat{V}_{1}\right) d \tau \\
& -\frac{n^{2}}{\cosh ^{2}(n \tau)}\left(q_{0}^{-1} \int \hat{V}_{B} d \tau+\iint\left(\hat{K}_{2}+\hat{V}_{2}-\hat{K}_{1}-\hat{V}_{1}\right.\right. \\
& \left.\left.\left.-2 \hat{K}_{3}-2 \hat{V}_{3}\right) d \tau d \tau\right)-2 n \int \tanh (n \tau)\left(g_{\phi}(\tau)+g_{1}(\tau)\right) d \tau\right] .
\end{aligned}
$$

Again, we have a similar situation as discussed in the absence of the $H$-field case in (39), and this equation leads to the following condition on the arbitrary constants:

$$
n^{2}\left(1-4 \alpha^{\prime} c_{2}\right)-2 p^{2}-4 q^{2}=0 .
$$

In $\alpha^{\prime} \rightarrow 0$ limit, the initial condition of $n^{2}-2 p^{2}-4 q^{2}=0$ that appears in the solutions of one-loop $\beta$ functions can be recovered. But in two-loop order, this condition has been modified by a term in the order $\alpha^{\prime}$, and $n^{2}-2 p^{2}-4 q^{2} \neq 0$ is required for consistency in the solution of (81). Then, the remaining terms in (81) fix the correction of the lapse function, $\xi_{4}$.

Now, calculating the integrals in (74)-(78) gives the explicit forms of the first $\alpha^{\prime}$ correction of the scale factors, dilaton field, and $H$ field. Because of dense mathematical results, the final forms of $\xi_{i}, \xi_{4}, \phi_{1}$, and $\eta_{1}$ in two RSs of Gauss-Bonnet and $R^{2}$ are presented in Appendix B.

\section{EINSTEIN FRAME REPRESENTATION}

Having solved the two-loop $\beta$-function equations in the two cases of the vanishing $H$ field and presence of a timedependent $H$ field in Sec. III, we return to Einstein frame field equations (9) in order to study the cosmological implications of the $\alpha^{\prime}$-corrected solutions. As mentioned in Sec. II, in the $\alpha^{\prime}$ order of effective actions, there is a field redefinition ambiguity and a class of equivalent effective actions corresponding to the same $S$ matrix. The cosmological effects of the field redefinition have been studied with the constant dilaton in Ref. [34] and with a time-dependent dilaton in Ref. [35], where a generalized effective action obtained by the field redefinitions has been investigated. However, here, we consider the Gauss-Bonnet effective action (8). In the Einstein frame, regarding $T_{\mu}^{\mu}=\left(-\rho, P_{1}, P_{2}, P_{3}\right)$, the nonzero components of energy-momentum tensors (11)-(14) give the effective energy density and pressures based on (10). The Einstein frame metric, which is related to the string frame metric by the conformal transformation (7), is considered as

$$
d s^{2}=\tilde{g}_{\mu \nu} d x^{\mu} d x^{\nu}=-d \tilde{t}^{2}+\sum_{i}^{3} \tilde{a}_{i}^{2}\left(d x^{i}\right)^{2},
$$


where $\tilde{a}_{i}$ are the Einstein frame scale factors related to the string frame ones by $\tilde{a}_{i}^{2}=e^{\phi} a_{i}^{2}$, in such a way that up to the first order of $\alpha^{\prime}$ we have

$$
\tilde{a}_{i}^{2}=e^{\phi_{0}} a_{i 0}^{2}\left(1+\alpha^{\prime}\left(2 \xi_{i}+\phi_{1}\right)\right) .
$$

Also, the time element in (83) has been defined using the following relation:

$$
d \tilde{t}=e^{\frac{\phi}{2}} \sqrt{g_{00}} d t .
$$

In the previous section, we found the solutions in terms of the time coordinate $\tau$, which, using (26), has the following relation with the cosmic time $\tilde{t}$ :

$$
d \tilde{t}=a_{0}{ }^{3} e^{3 \phi_{0} / 2}\left(1+\alpha^{\prime}\left(\sum \xi_{i}+\xi_{4}+\frac{3}{2} \phi_{1}\right)\right) d \tau .
$$

The integration of the above expression and transformation from $\tau$ to $\tilde{t}$ are not straightforward in the solutions; hence, for investigating the behavior of the solutions, the time derivatives in physical quantities in the Einstein frame will be rewritten in terms of $\tau$ derivatives such as

$$
\begin{aligned}
\dot{\tilde{a}}_{i} \equiv \frac{d \tilde{a}_{i}}{d \tilde{t}} & =\tilde{a}^{-3}\left(\tilde{a}_{i 0}^{\prime}+\alpha^{\prime}\left(\tilde{a}_{i 0}\left(\xi_{i}+\frac{1}{2} \phi_{1}\right)\right)^{\prime}\right) \\
\ddot{\tilde{a}}_{i} \equiv \frac{d^{2} \tilde{a}_{i}}{d \tilde{t}^{2}} & =\tilde{a}^{-6}\left(\tilde{a}_{i 0}^{\prime \prime}-\tilde{a}_{i 0}^{\prime} \sum \ln \tilde{a}_{j 0}^{\prime}+\alpha^{\prime}\left[\left(\tilde{a}_{i 0}\left(\xi_{i}+\frac{1}{2} \phi_{1}\right)\right)^{\prime \prime}\right.\right. \\
& -\tilde{a}_{i 0}^{\prime}\left(\ln \left(1+\sum \xi_{j}+\xi_{4}+\frac{3}{2} \phi_{1}\right)\right)^{\prime} \\
& \left.\left.+\alpha^{\prime}\left(\tilde{a}_{i 0}\left(\xi_{i}+\frac{1}{2} \phi_{1}\right)\right)^{\prime} \sum \ln \tilde{a}_{j 0}^{\prime}\right]\right)
\end{aligned}
$$

Here and hereafter, the dot symbol stands for derivation with respect to $\tilde{t}$. Also, the second derivative of the averaged scale factor up to first order of $\alpha^{\prime}$ is given by

$$
\begin{aligned}
\ddot{\tilde{a}}= & \frac{d^{2}}{d \tilde{t}^{2}}\left(\tilde{a}_{1} \tilde{a}_{2} \tilde{a}_{3}\right)^{\frac{1}{3}}=\frac{1}{9}\left(\tilde{a}_{1} \tilde{a}_{2} \tilde{a}_{3}\right)^{\frac{1}{3}}\left(3 \sum \dot{\tilde{H}}_{i}-\left(\sum \tilde{H}_{i}\right)^{2}\right) \\
= & \tilde{a}^{-5}\left(\left(\ln \tilde{a}_{i 0}\right)^{\prime \prime}-\left(\ln \tilde{a}_{i 0}\right)^{\prime} \sum\left(\ln \tilde{a}_{i 0}\right)^{\prime}+\alpha^{\prime}\left[\left(\xi_{i}+\frac{1}{2} \phi_{1}\right)^{\prime \prime}\right.\right. \\
& -\left(\ln \tilde{a}_{i 0}\right)^{\prime}\left(\ln \left(1+\sum \xi_{j}+\xi_{4}+\frac{3}{2} \phi_{1}\right)\right)^{\prime} \\
& \left.\left.+\alpha^{\prime}\left(\xi_{i}+\frac{1}{2} \phi_{1}\right)^{\prime} \sum \ln \tilde{a}_{j 0}^{\prime}\right]\right) .
\end{aligned}
$$

In Eq. (86), if the coefficient term of $d \tau$ is positive, $\tilde{t}$ will be an increasing function of $\tau$, and then $d \tau>0$ if and only if $d \tilde{t}>0$. In this sense, the early and late behaviors of solutions can be investigated in the $\tau \rightarrow 0$ and $\tau \rightarrow \infty$ limits.

The solutions of $\beta$-function equations for the metric, dilaton field, and $B$ field contain integrating constants that are allowed to be any real number, provided that some of them satisfy the initial conditions of (40) in the vanishing
$H$-field case and (82) in the presence of the $H$ field. It turns out that the constants appearing in the zeroth-order solutions affect the general behavior of solutions, while the constants of the first $\alpha^{\prime}$ corrections influence the early-time behavior. As a matter of fact that it is not convenient to predict the cosmological behavior of solutions without selecting some values for these constants, we are going to investigate the features of the obtained solutions with some chosen set of arbitrary constants. In this regard, besides the $\beta$-function prescribed initial conditions of (40) and (82), the other conditions that can be demanded from the cosmological point of view to be imposed on the obtained solutions are the positive sign of the coefficient term of $d \tau$ in (86), satisfying the energy condition $\rho^{\text {(eff) }}>0$, and having no singularity corresponding to the vanishing of scale factors in the future. Also, it should be noticed that the calculation of $\alpha^{\prime}$ corrections is trusted as long as the string coupling at tree level of the string interaction is weak, i.e., $g_{s} \ll 1$. Hence, the reliable area of solutions may be affected by selected parameters.

\section{A. Evolution with vanishing $\boldsymbol{H}$ field}

In the absence of the $H$ field, the solutions of two-loop $\beta$-function equations were found in Sec. III A. In the Einstein frame, according to (11), we have the dilaton field and charge deficit term $\Lambda$ contributions to the effective energy density and pressures up to first order of $\alpha^{\prime}$ as follows:

$$
\begin{aligned}
\rho^{(\phi)} & =\frac{1}{4}\left(\dot{\phi}^{2}-2 \Lambda e^{-\phi}\right) \\
& =\frac{1}{4}\left(\dot{\phi}_{0}^{2}-2 \Lambda e^{-\phi_{0}}+\alpha^{\prime}\left(2 \dot{\phi_{0}} \dot{\phi_{1}}+2 \Lambda e^{-\phi_{0}} \phi_{1}\right)\right), \\
P_{i}^{(\phi)} & =\frac{1}{4}\left(\dot{\phi}^{2}+2 \Lambda e^{-\phi}\right) \\
& =\frac{1}{4}\left(\dot{\phi}_{0}^{2}+2 \Lambda e^{-\phi_{0}}+\alpha^{\prime}\left(2 \dot{\phi_{0}} \dot{\phi_{1}}-2 \Lambda e^{-\phi_{0}} \phi_{1}\right)\right) .
\end{aligned}
$$

Also, $T_{\mu \nu}^{(\mathrm{GB})}(13)$ gives

$$
\begin{aligned}
& \rho^{(\mathrm{GB})}=-3 \alpha^{\prime} e^{\phi} \dot{\phi} \tilde{H}_{1} \tilde{H}_{2} \tilde{H}_{3}, \\
P_{1}^{(\mathrm{GB})}= & \alpha^{\prime} e^{\phi}\left(\tilde{H}_{2} \tilde{H}_{3}\left(\ddot{\phi}+\dot{\phi}^{2}\right)+\dot{\phi}\left(\tilde{H}_{2} \dot{\tilde{H}}_{3}+\tilde{H}_{3} \dot{\tilde{H}}_{2}\right.\right. \\
& \left.+\tilde{H}_{2} \tilde{H}_{3}\left(\tilde{H}_{2}+\tilde{H}_{3}\right)\right), \\
P_{2}^{(\mathrm{GB})}= & \alpha^{\prime} e^{\phi}\left(\tilde{H}_{1} \tilde{H}_{3}\left(\ddot{\phi}+\dot{\phi}^{2}\right)+\dot{\phi}\left(\tilde{H}_{1} \dot{\tilde{H}}_{3}+\tilde{H}_{3} \dot{\tilde{H}}_{1}\right.\right. \\
& \left.+\tilde{H}_{1} \tilde{H}_{3}\left(\tilde{H}_{1}+\tilde{H}_{3}\right)\right), \\
P_{3}^{(\mathrm{GB})}= & \alpha^{\prime} e^{\phi}\left(\tilde{H}_{2} \tilde{H}_{1}\left(\ddot{\phi}+\dot{\phi}^{2}\right)+\dot{\phi}\left(\tilde{H}_{2} \dot{\tilde{H}}_{1}+\tilde{H}_{1} \dot{\tilde{H}}_{2}\right.\right. \\
& \left.+\tilde{H}_{2} \tilde{H}_{1}\left(\tilde{H}_{2}+\tilde{H}_{1}\right)\right),
\end{aligned}
$$

where the $\tilde{H}_{i}$ are Einstein frame Hubble parameters defined by $\tilde{H}_{i}=\frac{d}{d t} \ln \tilde{a}_{i}[42]$. It should be noted that for investigating 
the solutions up to first order of $\alpha^{\prime}$ only the zeroth order of $\tilde{H}_{i}$ and $\phi$ contributes in $T_{\mu \nu}^{(\mathrm{GB})}$ and $T_{\mu \nu}^{\left(\mathrm{B}_{2}\right)}$.

The $\alpha^{\prime}$-corrected string frame scale factors, lapse function, and dilaton field introduced in (23)-(25) have been found in the forms of (32), (33), and (A1)-(A3) in the absence of the $H$ field. The leading-order string coupling, in this case, is given by

$$
g_{s}=\mathrm{e}^{-\phi_{0}}=\frac{\sqrt{\Lambda} L_{1} L_{2} L_{3}}{n} \cosh (n \tau) \mathrm{e}^{\sum q_{i} \tau} .
$$

Aiming at considering only the $\alpha^{\prime}$ corrections, the string coupling is required to be weak. In early $\tau$, it can be achieved by setting the $L_{i}$ constants at least of order $\sqrt{\alpha^{\prime}}$. Then, with $\sum q_{i}<0$, the weak string coupling condition is satisfied in all times with the proper selection of $n$, but with $\sum q_{i}>0$, the $g_{s}$ may leave the weak coupling limit as time goes on. Here, we present two examples with isotropic and anisotropic parametrization. The constants appearing in the solutions are $q_{i}, n, l_{i}, L_{i}, Q_{1}$, and $Q_{2}$, where $q_{i}, n$, and $Q_{2}$ have to satisfy the initial condition (40).

For an isotropic example, we set $q_{i}=-2, n=1$, $L_{i}=\sqrt{\alpha^{\prime}}, l_{i}=-10, r_{i}=0, Q_{1}=500$, along with using the (40) condition for fixing $Q_{2}$. Here, the string frame curvature that starts in the $R \alpha^{\prime} \gtrsim 1$ limit is increasing, and the string coupling is weak and decreasing. These imply that the $\alpha^{\prime}$-corrected solutions are valid and important even in late times and the string loop correction can be ignored. This example has positive but decreasing $\dot{\tilde{a}}$ and $\ddot{\tilde{a}}$. Effectively, the energy density is decreasing, and the pressure is negative and increasing. Also, the strong energy condition is violated by $\rho^{(\mathrm{eff})}+3 P^{(\mathrm{eff})}<0$, where the null energy condition $\rho^{\text {(eff) }}+P^{(\text {eff })} \geq 0$ is satisfied except in a short range of time near $\tau=0$. Hence, this example describes an accelerated expanding Universe with avoidance of initial singularity related to violation of strong energy condition. The phantom phase, which by definition satisfies the $P^{(\text {eff })}<-\rho^{\text {(eff) }}$ with the equation of state parameter $w$ less than -1 , is transient in early time.

Having found no compatible example with $\sum q_{i}<0$ in the anisotropic case with preserving the signature of metric (83) and the energy condition of $\rho^{\text {(eff) }}>0$, we set $q_{1}=1.1$, $q_{2}=1.5, q_{3}=2, n=2.4, l_{i}=1, r_{i}=0$, and $L_{i}=20 \sqrt{\alpha^{\prime}}$. The $Q_{2}$ is fixed by initial condition (40). This example gives positive and increasing $\dot{\tilde{a}}_{i}, \ddot{\tilde{a}}_{i}$, and $\ddot{\tilde{a}}$ defined in (87), (88), and (89). Furthermore, the $P_{i}^{(\text {eff })}$ are negative with violating the strong and null energy conditions. It is worth mentioning that as time goes on in this parametrization, accompanied by the unbounded growth of curvature that has started in the $R \alpha^{\prime} \gtrsim 1$ limit, the string coupling $g_{s}$ keeps growing until leaving the weak coupling limit. Reaching the strongly coupled high curvature phase signals the entering of the system into the full M-theory regime $[43,44]$. Nevertheless, this given solution is valid as long as the $g_{s}$ is sufficiently weak in early $\tau$ and describes an accelerated expansion in all directions with the avoidance of initial singularity and behaves as a phantom with $w_{i}<-1$. The existence of the $w<-1$ region opens up the possibility of the so-called big-rip singularity, which has been classified in four classes, occurring [45-48]. Noting the scale factors and dilaton field given by (32), (33), and (A1)-(A3), no finite-time singularity appears in the scale factors, derivatives of Hubble parameters, dilaton field (and their derivatives), nor, consequently, according to (90)-(95), the pressures and energy density. Exhibiting no sudden future time divergence by these quantities, which is also verified by their plots, implies that none of the four types of big-rip singularities occurs.

\section{B. Evolution with the nonvanishing $\boldsymbol{H}$ field}

Considering a spatially homogeneous time-dependent $B$ field with the field strength tensor of type (41), the solutions of two-loop $\beta$-function equations have been found in Sec. III B. A usual effect of this type of $H$ field is an anisotropic evolution in spatial directions. Also, the contribution of the $B$ field brings up a RS dependence on the two loop-order $\beta$-function equations and consequently in their solutions. We have considered two special RSs of $R^{2}$ and Gauss-Bonnet corresponding to the RS parameters of $f=1$ and $f=-1$, respectively. In the Gauss-Bonnet scheme, with the $H$ field of the form (41), the components of energy-momentum tensors (12) and (14) recast the following forms:

$$
\begin{aligned}
& \rho^{\left(B_{1}\right)}=-P_{1}^{\left(B_{1}\right)}=-P_{2}^{\left(B_{1}\right)}=P_{3}^{\left(B_{1}\right)}=\frac{1}{4} A^{2} \tilde{a}_{1}^{2} \tilde{a}_{2}^{2} e^{2 \phi}, \\
& \rho^{\left(B_{2}\right)}=\frac{\alpha^{\prime}}{64} A^{2} \tilde{a}_{1}^{2} \tilde{a}_{2}^{2} e^{-\phi}\left(\frac{38}{3} A^{2} \tilde{a}_{1}^{2} \tilde{a}_{2}^{2} e^{-2 \phi}+15 \tilde{H}_{1} \tilde{H}_{2}\right. \\
& -8\left(\tilde{H}_{1}+\tilde{H}_{2}\right)\left(\tilde{H}_{3}+3 \dot{\phi}\right)+32\left(\tilde{H}_{2}^{2}+\tilde{H}_{1}^{2}\right) \\
& \left.+4 \dot{\phi}^{2}+48\left(\dot{\tilde{H}}_{1}+\dot{\tilde{H}}_{2}\right)\right) \text {, } \\
& P_{1}^{\left(B_{2}\right)}=\frac{\alpha^{\prime}}{64} \tilde{a}_{1}^{2} \tilde{a}_{2}^{2} e^{-\phi}\left(-\frac{26}{3} A^{4} \tilde{a}_{1}^{2} \tilde{a}_{2}^{2} e^{-2 \phi}+8 \dot{A}^{2}+\left(72 \dot{\phi}^{2}\right.\right. \\
& -8\left(6 \tilde{H}_{1}-\tilde{H}_{2}\right) \dot{\phi}-16 \tilde{H}_{1}^{2}-\left(31 \tilde{H}_{2}+32 \tilde{H}_{3}\right) \tilde{H}_{1} \\
& -80 \tilde{H}_{2}^{2}-8 \ddot{\phi}-24 \tilde{H}_{2} \tilde{H}_{3}-64 \dot{\tilde{H}}_{1}-48 \dot{\tilde{H}}_{2} \\
& \left.\left.-24 \dot{\tilde{H}}_{3}\right) A^{2}+16\left(3 \tilde{H}_{1} \dot{A}+2 \tilde{H}_{2} \dot{A}-3 \dot{\phi} \dot{A}+\ddot{A}\right) A\right) \text {, } \\
& P_{2}^{\left(B_{2}\right)}=P_{1}^{\left(B_{2}\right)}(1 \leftrightarrow 2), \\
& P_{3}^{\left(B_{2}\right)}=\frac{-\alpha^{\prime}}{192} A^{2} \tilde{a}_{1}^{2} \tilde{a}_{2}^{2} e^{-\phi}\left(10 A^{2} \tilde{a}_{1}^{2} \tilde{a}_{2}^{2} e^{-2 \phi}+36 \dot{\phi}^{2}-3 \tilde{H}_{1} \tilde{H}_{2}\right) \text {. }
\end{aligned}
$$

The contributions of the dilaton field and Gauss-Bonnet term in the effective energy-momentum tensor are the same 
as given by (90)-(95). Again, only the zeroth order of $\tilde{a}_{i}$, $\tilde{H}_{i}, A$, and $\phi$ will be effective in the components of $T_{\mu \nu}^{(\mathrm{GB})}$ and $T_{\mu \nu}^{\left(B_{2}\right)}$. The $H$ field in this class brings about anisotropic pressures, which means that it acts like an anisotropic fluid. Its contribution to the pressures in the zeroth order of $\alpha^{\prime}$, given by (98), is negative in the $x^{1}$ and $x^{2}$ directions and positive in the $x^{3}$ direction, but the signs in the first order of $\alpha^{\prime}$ may be affected by selected values for the arbitrary constants of solutions.

In Sec. III B, the solutions of two-loop $\beta$-function equations gave the $\alpha^{\prime}$-corrected string frame scale factors, lapse function, dilaton field, and $H$ field of (23)-(25) and (57) in the forms of (62)-(64) and (74)-(78), where the final forms of correction terms after performing the integrals are presented in Appendix B through (B3)(B12). Regarding the obtained scale factors, it turns out that the anisotropy is inevitable with the $H$ field in this considered class. Here, we are going to investigate the behavior of solutions by choosing some values for arbitrary constants in two RSs and study the feature of energymomentum tensor components of the Gauss-Bonnet scheme. The constants appearing in the solutions are $q, p, n, b, c_{1}, c_{2}, r_{i}, \varphi_{0}$, and $L_{3}$, where the $p, q, n$, and $c_{2}$ must satisfy the initial condition (82).

According to (64), the leading-order string coupling is given by

$$
g_{s}=e^{-\phi_{0}}=L_{3} b^{-1} \sqrt{\Lambda} e^{p \tau} .
$$

Its value in the origin of $\tau$ can be set to be sufficiently small, for instance, by letting the $L_{3}$ and $b^{-1}$ constants be in order $\sqrt{\alpha^{\prime}}$. The behavior of $g_{s}$ depends on the sign of $p$, in such a way that with $p \leq 0$ the weak coupling condition is always satisfied, but with $p>0$, it increases going forward in time and may leave the weak coupling limit.

In the $R^{2}$ scheme, as an example, the set of $q=3$, $p=-2, n=3, L_{3}=2 \sqrt{\alpha^{\prime}}, b^{-1}=2 \sqrt{\alpha^{\prime}}, \varphi_{0}=12, c_{1}=1$, and $r_{i}=0$ can be chosen. Investigating $\dot{\tilde{a}}_{i}$ shows that this example is expanding in all direction in early times and then turns to Kasner-type expanding, i.e., expanding in two directions and contracting in one direction with $\dot{\tilde{a}}_{1}<0$, followed by a phase of expansion in all directions. Moreover, the behavior $\ddot{\tilde{a}}_{i}$ is as follows: $\ddot{\tilde{a}}_{1}$ is negative in early times and then turns to be positive, the $\ddot{\tilde{a}}_{2}$ is positive forever, and the $\ddot{\tilde{a}}_{3}$ has behavior similar to the first direction but leaves the negative phase earlier [49]. Also, the first and second derivatives of the averaged scale factor (89) are positive, which shows that the expansion is accelerated.

In the Gauss-Bonnet scheme, for example, the parametrization of $q=1, \quad p=3.15, \quad n=5, \quad L_{3}=\sqrt{\alpha^{\prime}}$, $b^{-1}=\sqrt{\alpha^{\prime}}, \varphi_{0}=300, r_{3}=350$, and $c_{1}=r_{2}=r_{2}=0$, with using (73) to fix $c_{2}$, is capable of preserving $\rho^{\text {(eff) }}>0$, preventing the vanishing of scale factors, and making the coefficient of $d \tau$ in (86) be positive. This example starts expanding in all directions and then becomes contracting in the first and third directions, where the $\dot{\tilde{a}}_{1}$ leaves the negative area earlier than $\dot{\tilde{a}}_{3}$, and then becomes FRW-type expansion along all directions. In addition, investigating the behavior of $\ddot{\tilde{a}}_{i}(88)$ shows that $\ddot{\tilde{a}}_{1}$ and $\ddot{\tilde{a}}_{2}$ are negative at first and then become positive and keep increasing, but $\ddot{\tilde{a}}_{3}$ is negative and decreasing. Also, the averaged scale factor has $\ddot{\tilde{a}}>0$, which implies that the evolution of the model is accelerated. Furthermore, $P_{1}^{\text {(eff) }}$ and $P_{2}^{(\text {eff })}$ start negatively and keep increasing to become positive, where $P_{3}^{\text {(eff) }}$ is negative and decreasing. Effectively, the strong energy condition $\rho^{\text {(eff) }}+\sum P_{i}^{\text {(eff) }}>0$ is violated, and hence the initial singularity is avoided. Also, the null energy condition is violated in the third direction so the timedependent equation of state parameter in the third direction is $w_{3}<-1$. However, investigating $\tilde{a}_{i}, \dot{\tilde{H}}_{i}, \rho^{\text {(eff) }}$, and $P^{\text {(eff) }}$ shows that there is no evidence of big-rip singularity occurrence corresponding to a sudden divergence in these quantities. It is worth mentioning that the $g_{s}$, starting in the weak coupling limit in early time, evolves toward the strong coupling because the $p$ is positive here. Hence, as a matter of fact that curvature and $g_{s}$ show unbounded growth, the calculation of $\alpha^{\prime}$ corrections is no longer valid in $\tau>p^{-1} \ln \left(\frac{b}{L_{3} \sqrt{\Lambda}}\right)$, when the condition of $g_{s} \ll 1$ is violated and Universe enters the non perturbative regime of the $\mathrm{M}$ theory.

\section{CONCLUSION}

The higher-derivative corrections are introduced to the string effective action when the equivalence between field equations and higher-loop $\sigma$-model $\beta$ functions is considered. Aimed at presenting noncritical Bianchi type I string cosmology solutions, we have solved the $\beta$-function equations in the presence of central charge deficit term $\Lambda$. Being of order $\alpha^{\prime-1}$, the $\Lambda$ term resulted in the leadingorder string curvature in the high curvature limit of $R \alpha^{\prime} \gtrsim 1$, which requires the consideration of higher-order $\beta$-function equations and consequently including the $\alpha^{\prime}$ corrections in the effective action. The other type of effective action modification, i.e., the stringy loop corrections, have been assumed to be negligible, which is reliable where the leading-order sting coupling is weak, i.e., $g_{s} \ll 1$.

Considering the two-loop (order $\alpha^{\prime}$ ) $\beta$-function equations with $\Lambda \neq 0$ in the two cases of a vanishing and nonvanishing $H$ field, we have calculated their solutions by implementing a perturbation series expansion up to first order of $\alpha^{\prime}$ on the background fields. The solutions provided an $\alpha^{\prime}$-corrected string frame metric, dilaton field, and $H$ field. Then, to study the cosmological implications of solutions, the corresponding solutions in the Einstein frame have been obtained by performing a conformal 
transformation on the metric. Also, the Einstein frame effective action has been considered to include the GaussBonnet term coupled to the dilaton field, because from the string theory point of view, the Gauss-Bonnet combination is indistinguishable from the other quadratic curvature corrections. In this sense, the effective energy-momentum tensor in the Einstein frame field equations contains the contributions of the Gauss-Bonnet term, dilaton, $\Lambda$, and $H$ field.

For investigating the detailed behaviors of the $\alpha^{\prime}$ corrected background field solutions and the effective energy density and pressures, we have considered some set of values for the arbitrary constants appearing in the solutions. These constants are allowed to be any real number, provided that some of them satisfy the initial condition. Particularly, preserving the energy condition $\rho^{\text {(eff) }}>0$ and avoiding the singularity in the metric caused by vanishing scale factors have been demanded in selecting the constants. Without the contribution of the $H$ field, the solutions are not necessarily anisotropic, and two examples with choosing isotropic and anisotropic parametrization were discussed. In the isotropic case, an example describing the accelerated expanding Universe with a transient phantom phase in early time was presented. In the offered anisotropic example, the dilaton field starts from weak coupling in early time and evolves to the strong coupling regime as time goes on. Hence, the calculation of $\alpha^{\prime}$ corrections is valid only in sufficiently small times with $g_{s} \ll 1$, where the given example describes an accelerated expansion that crosses the phantom phase $w_{i}<-1$ with violating the null energy condition in all directions. In addition, in the presence of a time-dependent $H$ field of which the $H_{012}$ component was considered to be nonzero, the solutions appeared to be inevitably anisotropic. In this case, the presented example with the chosen set of constants describes an accelerating model evolving from a Kasner-type phase to FRW-type expansion in all directions along with violating the null energy condition in the third direction. Its valid cosmological era is limited to early times by the growing of $g_{s}$.

It is worth mentioning that the conformal invariance condition prescribes including the whole $\alpha^{\prime}$-correction series. Especially, working at the high curvature limit, all higher orders of $\alpha^{\prime}$ corrections certainly become important. Nevertheless, aimed at finding a pattern given by including the corrections, we restricted our discussion to the first-order $\alpha^{\prime}$ (two-loop) $\beta$-function equations as the solutions of the first-order $\alpha^{\prime}$-corrected string effective action at zeroth order in the string coupling. Even in the first order, the corrections have been capable of i) excluding the initial singularity in the regime of violation of the strong energy condition and ii) describing the accelerated expansion of the Universe. However, as time passes, the validity of examples may be restricted by the growing of the string coupling and passing the weak coupling limit. Also, in the trustable area, the phantom phase may appear where the $w$ becomes less than -1 , but there is no big-rip, which is indicated by the finite future time divergence in scale factors, energy density, pressures, or time derivative of Einstein frame Hubble parameters [45,46].

The Gauss-Bonnet model coupled to a dynamical scalar field with a non-negative potential on FRW space-times has long been known to have nonsingular cosmological solutions by allowing the violations of both the null and the strong energy conditions [26]. Recently, the dark energy scenario has been investigated in this model, in which phantom phases have been predicted [38]. The $\Lambda$ term in the Einstein frame effective action (8) takes the form of a potential of type $V(\phi)=V_{0} e^{-\phi}$, where the $V_{0}$ is assigned to be negative in $D<26$-dimensional string theory. The vanishing $H$-field case presented in Secs. III A and IV A, which is described by the effective action similar to that of the Einstein-scalar-Gauss-Bonnet model [38] but with a negative potential, possesses the $w<-1$ phase in the investigated $\alpha^{\prime}$-corrected solutions, but the described Universe does not seem to reach a big-rip singularity. Also, it has been shown in Refs. [50,51] that bouncing solutions, which have a connecting phase between a contraction and an expansion period, are not allowed in the isotropic flat FRW Universe in the Einstein-scalarGauss-Bonnet model. The presented examples in Sec. IVA show the similar feature in both the isotropic and anisotropic cases, since they have no contraction phase and correspondingly no bounce phase.

The evolution with a nonvanishing homogeneous $H$ field with $H_{012} \neq 0$ has been studied in the low curvature phase in Refs. $[52,53]$ and in the high curvature phase including the $\alpha^{\prime}$ corrections in Ref. [27], in which the potential of the dilaton field or equivalently the $\Lambda$ term has been neglected, assuming the domination of the kinetic terms. However, this assumption made the valid cosmological era of the results be limited. We have seen that, particularly in the early $\tau, \Lambda$ is significant and cannot be ignored because none of the $R, H^{2}$, or $\dot{\phi}^{2}$ overcomes the $\Lambda$. However, in late $\tau$, the curvature and kinetic term of the dilaton field may dominate where the dynamical effect of the $H$ field becomes negligible.

Furthermore, the presented example with a nonvanishing $H$ field in Sec. IV B has no bounce phase in the $R^{2}$ scheme, but in the Gauss-Bonnet scheme, $\bar{H}$ crosses zero with $\dot{\bar{H}}>0$. Hence, the appearance of the bouncing solutions in string-inspired Einstein-scalar-Gauss-Bonnet with $B$-field contribution in the leading and first correction orders seems to be allowed but sensitive to the chosen RS.

\section{APPENDIX A: EXPLICIT SOLUTIONS OF VANISHING $H$ FIELD CASE}

In this Appendix, the explicit forms of the first $\alpha^{\prime}$ corrections of metric and dilaton field, introduced in 
(23)-(25), are presented. After performing the integrals in (36), (37), and (39) with using the (32) and (33) solutions, we obtain

$$
\begin{aligned}
\xi_{i}= & -\frac{\Lambda q_{i}^{2}}{2 n^{3}}\left(\frac{1}{2 n}\left(n^{2}+\sum q_{i}^{2}\right)\left(\cosh ^{2}(n \tau)+n^{2} \tau^{2}\right)+q_{i}(\cosh (n \tau) \sinh (n \tau)+n \tau)-\tau^{2} n^{3}\right)+q_{i} \int \xi_{4} d \tau+l_{i} \tau+r_{i} \\
\phi_{1}= & Q_{1} \tanh (n \tau)+Q_{2}(n \tau \tanh (n \tau)-1)-\left(n \tanh (n \tau)+\sum q_{i}\right) \int \xi_{4} d \tau+\frac{\Lambda}{4 n^{4}}\left[-\mathrm{e}^{-n \tau} \sum\left(q_{i}^{2}\left(n^{2}-\sum q_{j}^{2}\right) \tau\right.\right. \\
& \left.-4 q_{i}^{3}\right) \tanh (n \tau) n \sum q_{i}^{3}\left(2 \cosh ^{2}(n \tau)-3\right)+n\left(4 \sum\left(n q_{i}^{3}+q_{i}^{4}\right)+6 \sum q_{i<j} q_{j}\right) \tanh (n \tau) \tau \\
& +\left(\sum\left(n^{2} q_{i}^{2}+q_{i}^{4}\right)+\sum_{i<j} q_{i} q_{j}\right)\left((\cosh (n \tau))^{2}-2\right)-n \sum q_{i}^{2}\left(n^{2}-\sum q_{i}^{2}\right)(n \tau \tanh (n \tau)-2) \tau \\
& \left.+\frac{n}{\cosh (n \tau)}\left(\left(\sum q_{i}^{2}\left(n^{2}-\sum q_{j}^{2}\right) \tau+4 n^{2} \sum r_{i}-2 \sum q_{i}^{3}\right)\left((n \tau \tanh (n \tau)-1) \mathrm{e}^{-n \tau}-\frac{n \tau}{\cosh (n \tau)}\right)\right)\right]+\varphi_{0}
\end{aligned}
$$

where the $l_{i}$ and $\varphi_{0}$ are integrating constants, and then from (39), we have

$$
\begin{aligned}
\xi_{4}= & \frac{3 \Lambda}{4 n^{3}}\left[\frac{1}{3} n\left(\sum\left(n^{2} q_{i}^{2}+q_{i}^{4}\right)+\sum_{i<j} q_{i}^{2} q_{j}^{2}\right) \cosh (2 n \tau)+\frac{2}{3} n^{2} \sum q_{i}^{3} \sinh (2 n \tau)-\frac{8}{3} n\left(\sum\left(n^{2} q_{i}^{2}+2 q_{i}^{4}\right)\right.\right. \\
& \left.+\sum_{i \neq j} q_{i} q_{j}^{3}+\frac{1}{2} \sum_{i<j} q_{i}^{2} q_{j}^{2}\right) \cosh ^{2}(n \tau)-\frac{20}{15}\left(\sinh (n \tau)\left(\left(5 \sum q_{i}^{3}+\sum_{i \neq j} q_{i} q_{j}^{2}\right) n^{2}+\left(\sum q_{i}\right)^{2} q_{j}\right) \cosh (n \tau)\right) \\
& -\frac{2}{3} n\left(\sum\left(q_{i}^{2} \tau n^{3}-\left(2 q_{i}^{3} \tau+4 q_{i} \sum r_{j}+3 q_{i}^{2}\right) n^{2}\right)-2 \tau \sum_{i \neq j} q_{i} q_{j}^{2} n^{2}+\left(\sum q_{i}^{2}\right)^{2}\left(-n \tau+\left(2 \tau\left(\sum q_{j}\right)-1\right)\right)\right. \\
& +\frac{4}{3} \tanh (n \tau) n^{2}\left(\sum_{i}\left(\left(q_{i}^{2} \tau+2 r_{i}\right) n^{2}-q_{i}^{3}-\tau q_{i}^{4}\right)-2 \tau \sum_{i \neq j} q_{i}^{2} q_{j}^{2}\right)+\frac{1}{\cosh ^{2}(n \tau)}\left(\frac { 2 } { 3 } n ^ { 2 } \left(\sum \left(\left(q_{i}^{2} \tau+2 r_{i}\right) n^{2}\right.\right.\right. \\
& \left.\left.\left.\left.-\tau q_{i}^{4}+q_{i}^{3}\right)-2 \tau \sum_{i \neq j} q_{i}^{2} q_{j}^{2}\right) \cosh (n \tau) \mathrm{e}^{-n \tau}+\tau n^{3}\left(\left(\sum\left(q_{i}^{2} \tau+4 r_{i}\right) n^{2}-\tau q_{i}^{4}-2 q_{i}^{3}\right)-2 \tau \sum_{i \neq j} q_{i}^{2} q_{j}^{2}\right)\right)\right] .
\end{aligned}
$$

\section{APPENDIX B: EXPLICIT SOLUTIONS OF NONVANISHING HOMOGENEOUS H FIELD CASE}

With $m=n$, the solution of $\phi_{1}(78)$ reads

$$
\phi_{1}=\frac{1}{n}\left[\tanh (n \tau) \int(n \tau \tanh (n \tau)-1) g_{\phi}(\tau) d \tau-(n \tau \tanh (n \tau)-1) \int \tanh (n \tau) g_{\phi}(\tau) d \tau\right]-p \int \xi_{4} d \tau+\varphi_{0}
$$

in which $g_{\phi}$ is simply given by

$$
g_{\phi}(\tau)=-\frac{2 n^{2}}{\cosh ^{2}(n \tau)}\left(\iint\left(\hat{K}_{3}+\hat{V}_{3}\right) d \tau d \tau-q_{0}^{-1} \int \hat{V}_{B} d \tau\right)+\rho
$$

In the following, the explicit forms of the first $\alpha^{\prime}$ corrections of the metric, dilaton field, and $H$ field in (23)-(25) and (57) are presented for the case of contribution of the $H$ field in the $\rightarrow$ class, considered in Sec. III B. After performing the integrals of (74)-(78) and for the RS of $R^{2}, f=1$, and Gauss-Bonnet, $f=-1$, with $m=n$, we obtain

$$
\begin{aligned}
\xi_{1}^{(\mathrm{GB})}= & c_{1} \tanh (n \tau)+c_{2}(n \tau \tanh (n \tau)-1)+\left(\frac{n}{2} \tanh (n \tau)-q\right) \int \xi_{4} d \tau+r_{1} \\
& +\frac{\Lambda p^{2}}{8 n^{2}}\left[\tau\left(\left(n^{2} \tau-2 n q \tau-2 n-4 q\right) \tanh (n \tau)-2 n+4 q\right)+L i_{2}\left(-\mathrm{e}^{2 n \tau}\right) \tanh (n \tau)\right. \\
& \left.+\frac{q}{n}(\cosh (2 n \tau))^{-2}\left(n \tau\left(\mathrm{e}^{-2 n \tau}+1\right) \tanh (n \tau)-2 n \tau+\mathrm{e}^{-2 n \tau}+1\right)+2 \ln \left(\mathrm{e}^{2 n \tau}+1\right)\right]+\left.F(\tau)\right|_{f=-1}
\end{aligned}
$$




$$
\begin{aligned}
\xi_{1}^{\left(R^{2}\right)}= & c_{1} \tanh (n \tau)+c_{2}(n \tau \tanh (n \tau)-1)+\left(\frac{n}{2} \tanh (n \tau)-q\right) \int \xi_{4} d \tau+r_{1} \\
& +\frac{\Lambda}{8 n^{2}}\left[( \operatorname { c o s h } ( 2 n \tau ) ) ^ { - 2 } \left(-\ln \left(\mathrm{e}^{2 n \tau}+1\right)\left(2 n^{2}-p^{2}+\left(n\left(p^{2} \tau+n\right) \tanh (n \tau)-p^{2}\right) \cosh (2 n \tau)\right.\right.\right. \\
& \left.-\left(2 \cosh ^{2}(n \tau)-1\right) \tau n p^{2} \tanh (n \tau)\right)+\frac{1}{n}\left(\left(\frac{1}{2} n\left(-2 \ln (2) n^{2}+n^{2}\left(p^{2} \tau^{2}+1\right)-2 p^{2} q n \tau^{2}+2 p^{2} q \tau\right) \tanh (n \tau)\right.\right. \\
& \left.-\frac{1}{2} p^{2}\left(n^{2} \tau-2 n q \tau-2 q\right)\right) \mathrm{e}^{-2 n \tau}+\tau n\left(n\left(\frac{1}{2} n p^{2} \tau-p^{2} q \tau+n^{2}\right) \tanh (n \tau)-\frac{1}{2} p^{2}(n-2 q)\right) \mathrm{e}^{2 n \tau} \\
& \left.\left.+n\left(-\ln (2) n^{2}+n^{3} \tau+\left(p^{2} \tau^{2}+\frac{1}{2}\right) n^{2}-2 p^{2} q n \tau^{2}+p^{2} q \tau\right) \tanh (n \tau)+2 n^{4} \tau-n^{2} p^{2} \tau+2 n^{3} \ln (2)-n^{3}+p^{2} q\right)\right) \\
& \left.-\tanh (n \tau) p^{2}\left(-L i_{2}\left(-\mathrm{e}^{2 n \tau}\right)+\tau(n \tau(n-2 q)+2 n+4 q)\right)\right]+\left.F(\tau)\right|_{f=1},
\end{aligned}
$$

where the $F(\tau)$ is given by

$$
\begin{aligned}
F(\tau)= & \frac{\Lambda}{32 n^{3}}\left(\operatorname { t a n h } ( n \tau ) \left(8 n^{3} \ln \left(\mathrm{e}^{2 n \tau}+1\right) \tau\left(\left(3 n^{2}+8 p^{2}+4 q^{2}\right) f+n^{2}+2 p^{2}+4 q^{2}\right)-4 n \tau\left(( n \tau + 2 ) n ^ { 2 } \left(3 n^{2}+8 p^{2}\right.\right.\right.\right. \\
& \left.\left.+4 q^{2}\right) f+\tau n^{5}+3 n^{4}+2 \tau\left(p^{2}+2 q^{2}\right) n^{3}+\left(4 p^{2} \tau q+6 p^{2}\right) n^{2}+8 p^{2} q^{2}+16 q^{4}\right)+4 n^{2} L i_{2}\left(-\mathrm{e}^{2 n \tau}\right)\left(f \left(3 n^{2}\right.\right. \\
& \left.\left.+8 p^{2}+4 q^{2}\right)+n^{2}+2 p^{2}+4 q^{2}\right)-\sinh (2 n \tau)\left(48 q^{4}+\left(16 \tau n^{3}+24 p^{2}\right) q^{2}-16 n^{2} p^{2} \tau q+3 n^{4}+6 n^{2} p^{2}\right) \\
& \left.+2 n \cosh (2 n \tau)\left(\left(n^{4}+2 n^{2} p^{2}+8 p^{2} q^{2}+16 q^{4}\right) \tau-12 n q^{2}+12 p^{2} q\right)+\frac{n^{4}(10 f+13)\left(2 n \tau-\mathrm{e}^{-2 n \tau}-1\right)}{\cosh ^{2}(n \tau)}\right) \\
& -(n \tau \tanh (n \tau)-1)\left(2 \cosh ^{2}(n \tau)\left(\left(n^{2}-4 q^{2}\right)^{2}+2 n^{2} p^{2}+8 p^{2} q^{2}\right)+16 \sinh (n \tau) p^{2} q \cosh (n \tau) n\right. \\
& \left.\left.-16 p^{2} \tau q n^{2}+4 n^{2} \ln (\cosh (n \tau))\left(\left(3 n^{2}+8 p^{2}+4 q^{2}\right) f+n^{2}+2 p^{2}+4 q^{2}\right)-n^{4} \tanh ^{2}(n \tau)(10 f+13)\right)\right) .
\end{aligned}
$$

Also, we have

$$
\begin{aligned}
& \xi_{2}=\xi_{1}+2 q \int \xi_{4} d \tau+\frac{\Lambda p^{2} q}{2 n^{3}}(\sinh (n \tau) \cosh (n \tau)+n \tau)+r_{2} \\
& \xi_{3}=p \int \xi_{4} d \tau+\frac{p^{2} \Lambda}{4 n^{4}}\left[\left(\frac{3}{2} n^{2}+p^{2}+2 q^{2}\right) \cosh ^{2}(n \tau)+2 \sinh (n \tau) \cosh (n \tau) n p\right. \\
& \left.+\left(\tau\left(f+\frac{1}{2}\right) n^{2}+p^{2} \tau+2 q^{2} \tau+2 p\right) n^{2} \tau\right]+r_{3} \\
& \phi_{1}^{(\mathrm{GB})}=\left.U(\tau)\right|_{f=-1}-p \int \xi_{4} d \tau+\frac{\Lambda p^{3}}{8 n}\left(\tanh (n \tau)\left(-n \tau^{2}-2 \tau+2 \tau \ln \left(1+\mathrm{e}^{2 n \tau}\right)+\frac{1}{n} L i_{2}\left(-\mathrm{e}^{2 n \tau}\right)\right)\right. \\
& \left.-\frac{2}{n}(n \tau \tanh (n \tau)-1) \ln (\cosh (n \tau))\right)+\varphi_{0}, \\
& \phi_{1}^{(R 2)}=\left.U(\tau)\right|_{f=1}-p \int \xi_{4} d \tau+\frac{\Lambda}{8 n^{2}}\left(\operatorname { t a n h } ( n \tau ) \left(( \operatorname { c o s h } ( n \tau ) ) ^ { - 2 } n \left(2 n^{2} \tau^{2}+4 n \ln (2) \tau+1 / 2+n \tau(2 \sinh (2 n \tau)-1\right.\right.\right. \\
& \left.\left.+2 \ln (2) \mathrm{e}^{2 n \tau}\right)+\frac{1}{2} \mathrm{e}^{-2 n \tau}\right)+\ln \left(1+\mathrm{e}^{2 n \tau}\right)\left(2 p^{2} \tau-\frac{3 n}{4 \cosh ^{2}(n \tau)}(2 n \tau+\sinh (n \tau))\right)+\frac{p^{2}}{n} L i_{2}\left(-\mathrm{e}^{2 n \tau}\right)-n \ln (2) \\
& \left.\left.-\frac{n^{2} \tau \mathrm{e}^{n \tau}}{2 \cosh (n \tau)}+\left(-n \tau^{2}-2 \tau\right) p^{2}+n^{2} \tau\right)+(n \tau \tanh (n \tau)-1)\left(\frac{2\left(n^{2}-\cosh ^{2}(n \tau) p^{2}\right) \ln (\cosh (n \tau))+n^{2}}{n \cosh ^{2}(n \tau)}\right)\right)+\varphi_{0},
\end{aligned}
$$


where the $U(\tau)$ is given by

$$
\begin{aligned}
U(\tau)= & \frac{\Lambda}{32 n^{3}}\left(\operatorname { t a n h } ( n \tau ) \left(\tau \left(11 n^{5} \tau+(-8 \tau+18) n^{4}+2 \tau\left(p^{2}+58 q^{2}+2\right) n^{3}+\left(\left(24 p^{3}-16 p^{2}-32 q^{2}+8 p\right) \tau+44 p^{2}\right.\right.\right.\right. \\
& \left.\left.+104 q^{2}+4\right) n^{2}+\left(-4 p^{4} \tau-8 p^{2} q^{2} \tau+16 p^{3}\right) n+24 p^{4}+80 p^{2} q^{2}+64 q^{4}+16 q^{2}\right)-n L i_{2}\left(-\mathrm{e}^{2 n \tau}\right)\left(\left(18 n^{2}+36 p^{2}\right.\right. \\
& \left.\left.+24 q^{2}\right) f+11 n^{2}+4 p^{2}+116 q^{2}+4\right)+\frac{1}{4 \cosh ^{2}(n \tau)}\left(4 f n^{3}\left(4 n p^{2} \tau^{3}+2 p^{2} \tau^{2}-34 n \tau+17\right)+8 n p^{2}\left(n^{2}+2 p^{2}\right.\right. \\
& \left.+4 q^{2}\right) \tau^{3}+4 p^{2}\left(n^{2}+8 n p+2 p^{2}+4 q^{2}\right) \tau^{2}-4\left(35 n^{3}+2 p^{3}\right) \tau+70 n^{2}+\left(8 f\left(p^{2} \tau^{2}+17 / 2\right) n^{3}+4 p^{2}\left(\left(n^{2}+2 p^{2}\right.\right.\right. \\
& \left.\left.\left.\left.+4 q^{2}\right) \tau-8 p\right) \tau n+70 n^{3}\right) \mathrm{e}^{-2 n \tau}\right)-2 n^{2} \tau \ln \left(1+\mathrm{e}^{2 n \tau}\right)\left(f\left(18 n^{2}+36 p^{2}+24 q^{2}\right)+11 n^{2}+4 p^{2}+116 q^{2}+4\right) \\
& +2\left(\left(-4 p^{4}-8\left(n^{2}+2 q^{2}\right) p^{2}+n^{4}+\left(32 q^{2}+1\right) n^{2}-16 q^{4}-4 q^{2}\right) \tau+6\left(4 p^{3}-n^{2}-2 p^{2}-4 q^{2}+p\right)\right) \cosh (2 n \tau) \\
& \left.+\frac{1}{n}\left((8 \tau-3) n^{4}+\left(-32 p^{3} \tau+8(2 \tau+3) p^{2}-8 p \tau-3+32(\tau-3) q^{2}\right) n^{2}+12\left(p^{4}+q^{2}\left(4 p^{2}+4 q^{2}+1\right)\right)\right) \sinh (2 n \tau)\right) \\
& -(n \tau \tanh (n \tau)-1)\left(-2 n \ln \left(1+\mathrm{e}^{2 n \tau}\right)\left(\left(18 n^{2}+36 p^{2}+24 q^{2}\right) f+11 n^{2}+4 p^{2}+116 q^{2}+4\right)+\tau\left(f n ^ { 2 } \left(9 n^{2} \tau\right.\right.\right. \\
& \left.\left.+64 p^{2}+48 q^{2}\right)+22 n^{4}-16 n^{3}+\left(4 p^{2}+232 q^{2}\right) n^{2}+\left(48 p^{3}-32 p^{2}-64 q^{2}+16 p\right) n-8 p^{4}-16 p^{2} q^{2}\right) \\
& +(\cosh (n \tau))^{-2} 4\left(n \tau^{2}+\tau\right) p^{4}+4(2 n \tau-1) p^{3}+2(n \tau+1)\left((2 f+1) n^{2}+4 q^{2}\right) \tau p^{2}+(-34 f-35) n^{3}+4\left(p^{2} \tau\right. \\
& \left.-p+\left((f+1 / 2) n^{2}+2 q^{2}\right) \tau\right) p^{2} \mathrm{e}^{-2 n \tau}+\frac{2}{n}\left(\cosh (2 n \tau)\left(n^{4}+\left(-8 p^{2}+32 q^{2}+1\right) n^{2}-16 q^{4}-4\left(\left(4 p^{2}+\right) q^{2}+p^{4}\right)\right)\right) \\
& \left.\left.+8\left(-4 p^{3}+n^{2}+2 p^{2}+8 q^{2}-p\right) \sinh (2 n \tau)\right)\right),
\end{aligned}
$$

$$
\eta^{(\mathrm{GB})}=\frac{b p^{2} \Lambda}{8 n^{3}}(\sinh (n \tau) \cosh (n \tau)+n \tau)
$$

$$
\eta^{\left(R^{2}\right)}=\frac{b \Lambda}{4 n}\left(-n^{2} \tau^{2}-2 \ln (2) n \tau-L i_{2}\left(-\mathrm{e}^{2 n \tau}\right)+\frac{p^{2} \sinh (2 n \tau)}{4 n^{2}}+\frac{1}{2} \frac{p^{2} \tau}{n}\right)
$$

Also, Eq. (81) gives

$$
\begin{aligned}
\xi_{4}^{(\mathrm{GB})}= & \frac{-\Lambda}{16 n^{2}\left(2 n^{2}-2 p^{2}-4 q^{2}\right)}\left[\ln \left(\mathrm{e}^{2 n \tau}+1\right)\left(7 n^{2}+40 p^{2}-92 q^{2}-4\right) n^{2}-\tau n^{2}\left(7 n^{4}+8 n^{3}+\left(38 p^{2}-92 q^{2}-4\right) n^{2}\right.\right. \\
& \left.+\left(-24 p^{3}+8(-q+2) p^{2}-8 p+32 q^{2}\right) n+4 p^{4}+8 p^{2} q^{2}\right)+\left(n^{4}+\left(-8 p^{2}+32 q^{2}+1\right) n^{2}-4 p^{4}-16 q^{4}-4 q^{2}\right. \\
& \left.-16 p^{2} q^{2}\right) \cosh (2 n \tau)+\left(4 n^{3}+\left(-16 p^{3}+8 p^{2}+16 q^{2}-4 p\right) n\right) \sinh (2 n \tau)+\frac{1}{2 \cosh ^{2}(n \tau)}\left(-2 n p^{2}\left(n^{2} \tau\right.\right. \\
& \left.-2 p^{2} \tau-4 q^{2} \tau+2 p-8 q\right) \mathrm{e}^{-2 n \tau}+\left(12 n^{4}+\left(16 p^{2}+224 q^{2}+8\right) n^{2}-64 q^{4}-32 q^{2}\right) \cosh ^{4}(n \tau)+32\left(-2 p^{3}\right. \\
& \left.+n^{2}+(q+2) p^{2}-p+4 q^{2}\right) n \sinh (n \tau) \cosh ^{3}(n \tau)+\left(6 n^{4}+\left(-48 p^{2}-216 q^{2}-8\right) n^{2}-48 p^{4}\right. \\
& \left.-96 p^{2} q^{2}\right) \cosh ^{2}(n \tau)-16 n p^{2} \sinh (n \tau)\left(n^{2} \tau-2 p^{2} \tau-4 q^{2} \tau+2 p+q\right) \cosh (n \tau)+n\left(\left(6 p^{2} \tau^{2}-7\right) n^{3}\right. \\
& \left.\left.-2 n^{2} \tau p^{2}-12 p^{2} \tau\left(p^{2} \tau+2 q^{2} \tau+2 p+4 q\right) n+4 p^{2}\left(p^{2} \tau+2 q^{2} \tau-p+4 q\right)\right)\right)+2\left(\operatorname { c o s h } ^ { 2 } ( n \tau ) \left(\left(n^{2}-4 q^{2}\right)^{2}\right.\right. \\
& \left.+2 p^{2}\left(n^{2}+4 q^{2}\right)\right)+8 \cosh (n \tau) n p^{2} q \sinh (n \tau)-8 p^{2} \tau q n^{2}+2 n^{2} \ln (\cosh (n \tau))\left(-2 n^{2}-6 p^{2}\right) \\
& \left.\left.-3 / 2 n^{4} \tanh ^{2}(n \tau)-2 n^{4}(\cosh (n \tau))^{-2}\right)\right],
\end{aligned}
$$




$$
\begin{aligned}
& \xi_{4}^{\left(R^{2}\right)}=\frac{-\Lambda(\cosh (n \tau))^{-2}}{1184\left(n^{2}-2 p^{2}-4 q^{2}\right)}\left[-592 \ln (\cosh (n \tau)) n^{2}-\left(\cosh (2 n \tau)\left(29 n^{2}-112 p^{2}+140 q^{2}+4\right)+333 n^{2}\right.\right. \\
& \left.-112 p^{2}+140 q^{2}+4\right) \ln \left(\mathrm{e}^{2 n \tau}+1\right)+\frac{1}{2 n^{2}}\left(\left(58 n^{5} \tau+(-16 \tau+2) n^{4}+\left(84 p^{2} \tau+280 q^{2} \tau+8 \tau\right) n^{3}\right.\right. \\
& \left.+\left(48 p^{3} \tau+-16(2 \tau+1) p^{2}+16 p \tau+2+64(-\tau+1) q^{2}\right) n^{2}-8 p^{4}-32 p^{2} q^{2}-32 q^{4}-8 q^{2}\right) \cosh (2 n \tau) \\
& +\left(n^{4}-4 n^{3}+\left(-8 p^{2}+32 q^{2}+1\right) n^{2}-4 p^{4}-16 p^{2} q^{2}-16 q^{4}-4 q^{2}\right) \cosh (4 n \tau)+8\left(-p^{4} \tau-4 p^{3}+n^{2}\right. \\
& \left.+2\left(-q^{2} \tau+1\right) p^{2}-p+4 q^{2}\right) n \sinh (2 n \tau)+4 n\left(-4 p^{3}+n^{2}+2 p^{2}+4 q^{2}-p\right) \sinh (4 n \tau)-8\left(17 n^{2} \tau-37 n q \tau\right. \\
& +p-37 q) n p^{2} \mathrm{e}^{-2 n \tau}-148 \tau n^{2} p^{2}(n-2 q) \mathrm{e}^{2 n \tau}+\left(888 n^{4}+\left(1184 p^{2}+16576 q^{2}+592\right) n^{2}-4736 q^{4}\right. \\
& \left.-2368 q^{2}\right) \cosh ^{4}(n \tau)+2368\left(-2 p^{3}+n^{2}+(q+2) p^{2}-p+4 q^{2}\right) n \sinh (n \tau) \cosh ^{3}(n \tau)+\left(-3108 n^{4}\right. \\
& \left.+\left(-3552 p^{2}-20720 q^{2}-592\right) n^{2}-3552 p^{4}-7104 p^{2} q^{2}\right) \cosh ^{2}(n \tau)+1184 n \sinh (n \tau)\left(3 n^{2} \tau+2 p^{2} \tau+4 q^{2} \tau\right. \\
& -2 p-q) p^{2} \cosh (n \tau)+608 n^{4} \ln (2)+666 n^{5} \tau+\left(-1764 p^{2} \tau^{2}-16 \tau+7115\right) n^{4}+\left(-224 p^{2} \tau+280 q^{2} \tau+8 \tau\right) n^{3} \\
& +\left(-1176 p^{4} \tau^{2}-2304 p^{3} \tau+\left(-2352 q^{2} \tau^{2}-1184 q \tau-32 \tau-8\right) p^{2}+16 p \tau+1+(-64 \tau+32) q^{2}\right) n^{2} \\
& \left.-8 p^{2}(-37 q+p) n-4\left(p^{4}+4 p^{2} q^{2}+4 q^{4}+q^{2}\right)\right)+148 n\left(\frac{1}{12 n^{3}} \cosh ^{4}(n \tau)\left(\left(n^{2}-4 q^{2}\right)^{2}+2 p^{2}\left(n^{2}+4 q^{2}\right)\right)-n\right. \\
& \left.\left.+\frac{1}{n^{2}} \cosh ^{2}(n \tau)\left(4 \sinh (n \tau) p^{2} q \cosh (n \tau)-4 p^{2} \tau q n+2 n \ln (\cosh (n \tau))\left(2 n^{2}+5 p^{2}+4 q^{2}\right)-\frac{23 n^{2}}{4} \tanh ^{2}(n \tau)\right)\right)\right] .
\end{aligned}
$$

[1] A. Tseytlin, Phys. Lett. B 178, 34 (1986).

[2] R. Metsaev and A. Tseytlin, Nucl. Phys. B293, 385 (1987).

[3] C. Callan, D. Friedan, E. Martinec, and M. Perry, Nucl. Phys. B262, 593 (1985).

[4] E. Fradkin and A. Tseytlin, Nucl. Phys. B261, 1 (1985).

[5] M. Gasperini, M. Maggiore, and G. Veneziano, Nucl. Phys. B494, 315 (1997).

[6] C. Hull and P. Townsend, Nucl. Phys. B301, 197 (1988).

[7] R. Brustein and G. Veneziano, Phys. Lett. B 329, 429 (1994).

[8] R. Metsaev and A. Tseytlin, Phys. Lett. B 191, 354 (1987).

[9] A. Tseytlin, Phys. Lett. B 176, 92 (1986).

[10] N. A. Batakis, Phys. Lett. B 353, 450 (1995).

[11] N. A. Batakis and A. A. Kehagias, Nucl. Phys. B449, 248 (1995).

[12] N. A. Batakis, Phys. Lett. B 353, 39 (1995).

[13] J. D. Barrow and K. E. Kunze, Phys. Rev. D 55, 623 (1997).

[14] J. D. Barrow and M. P. Dąbrowski, Phys. Rev. D 55, 630 (1997).

[15] J. D. Barrow and K. E. Kunze, Phys. Rev. D 56, 741 (1997).

[16] A. Tseytlin and C. Vafa, Nucl. Phys. B372, 443 (1992).

[17] A. Tseytlin, Classical Quantum Gravity 9, 979 (1992).

[18] D. S. Goldwirth and M. J. Perry, Phys. Rev. D 49, 5019 (1994).

[19] G. Exirifard and M. O’Loughlin, J. High Energy Phys. 12 (2004) 023.

[20] F. Naderi and A. Rezaei-Aghdam, Nucl. Phys. B923, 416 (2017).

[21] G. Niz and N. Turok, Phys. Rev. D 75, 126004 (2007).

[22] S. Kawai and J. Soda, Phys. Rev. D 59, 063506 (1999).
[23] E. J. Kim and S. Kawai, Phys. Rev. D 87, 083517 (2013).

[24] S. Tsujikawa, Ann. Phys. (Amsterdam) 15, 302 (2006).

[25] C. Cartier, E. Copeland, and M. Gasperini, Nucl. Phys. B607, 406 (2001).

[26] P. Kanti, J. Rizos, and K. Tamvakis, Phys. Rev. D 59, 083512 (1999).

[27] S. Foffa and M. Maggiore, Phys. Rev. D 58, 023505 (1998).

[28] M. Natsuume, Phys. Rev. D 50, 3949 (1994).

[29] J. D. Barrow and M. P. Dąbrowski, Phys. Rev. D 58, 103502 (1998).

[30] J. Maldacena and C. Nunez, Int. J. Mod. Phys. A 16, 822 (2001).

[31] K. Maeda, Phys. Rev. D 37, 858 (1988).

[32] K. Maeda and N. Ohta, Phys. Lett. B 597, 400 (2004).

[33] K. Bamba, Z.-K. Guo, and N. Ohta, Prog. Theor. Phys. 118, 879 (2007).

[34] K. Akune, K.-i. Maeda, and N. Ohta, Phys. Rev. D 73, 103506 (2006).

[35] K. Maeda, N. Ohta, and R. Wakebe, Eur. Phys. J. C 72, 1949 (2012).

[36] $\hat{R}_{\lambda \mu \nu}^{\kappa}=R_{\lambda \mu \nu}^{\kappa}-\frac{1}{2} \nabla_{\mu} H_{\nu \lambda}^{\kappa}-\frac{1}{2} \nabla_{\nu} H_{\mu \lambda}^{\kappa}+\frac{1}{4} H_{\nu \lambda}^{\gamma} H_{\mu \gamma}^{\kappa}-\frac{1}{4} H_{\mu \lambda}^{\gamma} H_{\nu \gamma}^{\kappa}$.

[37] A. Tseytlin, Phys. Lett. B 176, 92 (1986).

[38] S. Nojiri, S. D. Odintsov, and M. Sasaki, Phys. Rev. D 71, 123509 (2005).

[39] We have employed the series expansion of $\ln a_{i}{ }^{\prime}=\ln a_{i 0}{ }^{\prime}+\frac{\alpha^{\prime} \xi_{i}^{\prime}}{1+\alpha^{\prime} \xi_{i}}=\ln a_{i 0}{ }^{\prime}+\alpha^{\prime} \xi_{i}^{\prime}+\mathcal{O}\left(\alpha^{\prime 2}\right)$.

[40] Especially, if $\left.\left(\sum q_{i}\right)^{2}+\sum q_{i}^{2}\right)>\frac{n^{2}}{2}$, the Ricci is in the $R \alpha^{\prime} \gtrsim 1$ limit even in $\tau=0$. 
[41] E. J. Copeland, A. Lahiri, and D. Wands, Phys. Rev. D 51, 1569 (1995).

[42] Up to first order of $\alpha^{\prime}$, we have $\tilde{H}_{i}=\frac{d}{d \tilde{t}} \ln \tilde{a}_{i 0}+\alpha^{\prime} \frac{d}{d \tilde{t}}\left(\xi_{i}+\frac{1}{2} \phi_{1}\right)$.

[43] E. Witten, Nucl. Phys. B443, 85 (1995).

[44] P. Hoava and E. Witten, Nucl. Phys. B460, 506 (1996).

[45] B. M. Leith and I. P. Neupane, J. Cosmol. Astropart. Phys. 05 (2007) 019.

[46] S. Nojiri, S. D. Odintsov, and S. Tsujikawa, Phys. Rev. D 71, 063004 (2005).

[47] J. Barrow, Classical Quantum Gravity 21, L79 (2004).
[48] J. Barrow, G. Galloway, and F. Tipler, Mon. Not. R. Astron. Soc. 223, 835 (1986).

[49] Setting $q=-3$ just exchanges the behavior of first and second directions.

[50] L. Sberna and P. Pani, Phys. Rev. D 96, 124022 (2017).

[51] T. Kobayashi, Phys. Rev. D 94, 043511 (2016).

[52] E. J. Copeland, A. Lahiri, and D. Wands, Phys. Rev. D 50, 4868 (1994).

[53] E. J. Copeland, A. Lahiri, and D. Wands, Phys. Rev. D 51, 1569 (1995). 